Gruhn, D. H. - Publikationen der mexikanischen Germanistik im Wandel

\title{
Publikationen der mexikanischen Germanistik im
}

\section{Wandel (1971-2018): Deutsch als Fremdsprache}

\author{
[Publications in German Studiens in transformation (1971-2018): \\ German as a foreign language] \\ http://dx.doi.org/10.11606/1982-883724431
}

\section{Dorit Heike Gruhn ${ }^{1}$}

\begin{abstract}
This second part of a bibliographical study aims to outline the evolution of publications of German Studies in Mexico. We focus on the area of German as a foreign language, which consists of 480 titles found, starting in 1971. We are principally interested in authors and the institutions in which they work, media of publication, thematic priorities, languages of publication and the role of lecturers of the academic exchange services DAAD and OeaD. In addition, a comparative perspective will be adopted with regard to some of the data obtained in the first part of this study. Different quantitative phases of publications were found, the high point being from 2000 to 2009. In addition, there was a marked preponderance of a few authors, mostly linked to the UNAM. As a medium of publication, authors prefer the proceedings of congresses, followed by journal articles. Almost half of all works were issued by the Mexican Association of German Teachers (AMPAL). As for content, teaching practice topics and evaluation predominate. More than two thirds of the texts are written in German and by authors with L1 German. In recent years there has been a clear decrease in the number of publications.
\end{abstract}

Keywords: German Studies; German as a foreign language; Publications; Mexico.

Zusammenfassung: Der vorliegende Artikel bildet den zweiten Teil einer bibliographischen Studie, die die Entwicklung der Publikationen der mexikanischen Germanistik nachzeichnet, und umfasst den Bereich Deutsch als Fremdsprache. Aufgenommen wurden 480 Titel, beginnend mit dem Jahr 1971. Das Hauptinteresse der Analyse lag auf den Autoren und ihrer institutionellen Zugehörigkeit, den Publikationsmedien, thematischen Schwerpunkten sowie den Publikationssprachen und der Rolle der DAAD- und OeAD-Lektoren. Darüberhinaus wird auch eine vergleichende Perspektive eingenommen bezüglich einiger Daten, die aus dem ersten Teil der Studie hervorgingen, welcher sich auf die Germanistik im engeren Sinne, das heißt mit nicht sprachvermittlungsbezogenen Inhalten,

\footnotetext{
${ }^{1}$ Benemérita Universidad Autónoma de Puebla, Facultad de Lenguas, 24 Norte 2003 Colonia Humboldt, Puebla, Puebla 72370, México. E-mail: heike50@ hotmail.com. ORCID: 0000-0001-9024-5113
}

(cc) BY-NC

Pandaemonium, São Paulo, v. 24, n. 43, mai.-ago. 2021, p. 1-37 


\section{Gruhn, D. H. - Publikationen der mexikanischen Germanistik im Wandel}

bezieht. Sichtbar werden verschiedene quantitative Phasen der DaF-Veröffentlichungen, die ihren Höhepunkt in den Jahren 2000 bis 2009 finden. Es zeichnet sich auch eine wesentliche Konzentration weniger Autoren $\mathrm{ab}$, die mehrheitlich mit der UNAM in Verbindung stehen. Lektoren stellen einen hohen Anteil der Titel. Beliebtestes Publikationsmedium sind Kongressakten, gefolgt von Zeitschriftenartikeln, wobei die von dem Mexikanischen Deutschlehrerverband AMPAL herausgegebenen Schriften fast die Hälfte aller Einträge ausmachen. Inhaltlich dominieren Themen rund um Didaktik und Evaluierung. Mehr als zwei Drittel der Texte sind auf Deutsch verfasst, und diese mehrheitlich von Autoren mit L1 Deutsch. Das quanitative Publikationsaufkommens der letzten Jahre zeigt eine stark abfallende Tendenz.

Schlüsselwörter: Germanistik; Deutsch als Fremdsprache; Publikationen; Mexiko.

\section{Anfänge und Panorama der Lehre des Deutschen als}

\section{Fremdsprache in Mexiko}

Wer an der Aufarbeitung der Geschichte der Germanistik und deutschen Sprachlehre in Mexiko interessiert ist, kommt an den Schriften einer Forscherin nicht vorbei: Marianne Oeste de Bopp, Gründerin der universitären Germanistik in Mexiko Mitte der 50er-Jahre des 20. Jahrhunderts. Die nachfolgende historische Kurzübersicht stützt sich weitgehend auf ihre Arbeiten „El idioma alemán en México” (1954) und „Die Deutschen in Mexiko” (1979).

Die Ursprünge der institutionellen Lehre der deutschen Sprache in Mexiko sind anders als man vielleicht vermuten würde - nicht vorrangig an deutschsprachige Geistesgrößen der Literatur und Philosophie gebunden, sondern an den Bergbau. Sie beginnt mit ,studentischer Mobilität”, nämlich mit Mexikanern, die Ende des 18. Jahrhunderts an der Bergakademie in Freiberg/Sachsen studierten. 1792 gründet der Freiberg-Rückkehrer Fausto de Elhuyar noch unter dem Vizekönigreich Neuspanien das Colegio de Minería in MexikoStadt und bleibt - ebenso wie sein Nachfolger und Freiberg-Absolvent Andrés del Río - der deutschen Bergbauwissenschaft langfristig verbunden. Im Jahre 1833 wird im nun unabhängigen Mexiko ein Regierungsdekret zum öffentlichen Bildungswesen erlassen, das unter anderem explizit die Einrichtung einer Dozentur für Deutsch an diesem Colegio vorsieht. Gegen Mitte des 19. Jahrhunderts macht sich der Preuße Oloardo Hassey dort einen Namen, und zwar nicht nur als Sprachlehrer, sondern auch als Verfasser der ersten germanistischen Schriften in Mexiko: eine Grammatik und eine Geschichte der deutschen Literatur. Bald wird an einigen anderen Institutionen des Landes Deutsch angeboten, Hassey

Pandaemonium, São Paulo, v. 24, n. 43, mai.-ago. 2021, p. 1-37 
Gruhn, D. H. - Publikationen der mexikanischen Germanistik im Wandel

selbst findet ein weiteres Betätigungsfeld an der 1868 gegründeten Oberschule Escuela Nacional Preparatoria, welche der Universidad Nacional Autónoma de México (UNAM) eingegliedert ist und bis heute Deutsch auf dem Lehrplan führt (vgl. OESTE DE BoPP 1954: 165-170).

Infolge der Zunahme der deutschsprachigen Einwanderung nach Mexiko gegen Ende des 19. Jahrhunderts kommt es zur Gründung Deutscher Schulen in Privatträgerschaft. Vorreiter ist 1873 das Colegio Katthain in Mexiko-Stadt (das aber bald darauf wieder schließt), gefolgt von der Deutschen Schule in Mexiko-Stadt, 1894, der Deutschen Schule in Puebla, 1911, und der Deutschen Schule in Guadalajara, 1917; des Weiteren entsteht 1936 eine deutschsprachige Mennoniten-Schule in Ciudad Cuauhtémoc, Chihuahua (vgl. OESTE DE BopP 1979: 523; 563f.) $)^{2}$. Gegenwärtig (Dezember 2019) bestehen in Mexiko fünf deutsche Auslandsschulen (betreut von der Zentralstelle für das deutsche Auslandsschulwesen), sieben Fit-Schulen (Schulen des mexikanischen Bildungssystems, die das deutsche Sprachdiplom anbieten) und zwei DSD-Schulen (betreut vom Goethe-Institut, darunter die Schweizer Schule mit Zweigstellen in drei verschiedenen Städten). Darüber hinaus gibt es seit 2011 das Colegio Austriaco-Alemán in Querétaro, Qto., das nicht in das Netz der PASCH-Schulen ${ }^{3}$ eingebunden ist.

Deutschunterricht an sprach- bzw. geisteswissenschaftlichen Fakultäten und universitären Sprachzentren setzt nach dem Zweiten Weltkrieg gegen Mitte des 20. Jahrhunderts ein. Auftrieb erhält er 1966 durch die Gründung des Centro de Enseñanza de Lenguas Extranjeras $\left(\mathrm{CELE}^{4}\right)$ an der UNAM, wo noch im selben Jahrzehnt eine eigene Deutschabteilung aufgebaut wird. Das Modell findet Nachahmer, gegenwärtig haben landesweit die meisten autonomen ${ }^{5}$ und großen Privatuniversitäten ein Sprachenzentrum, an

\footnotetext{
${ }^{2}$ Die Auflistung der Deutschen Schulen erhebt keinen Anspruch auf Vollständigkeit. Weitere solcher Schulen bestanden vorübergehend.

3 Dabei handelt es sich um ein weltweites Netz von Schulen mit DaF-Unterricht, das Schulleitungen, Lehrkräften und Schülern Unterstützung in verschiedener Form bietet. Standorte in Mexiko: www.paschnet.de/de/pasch-schulen/weltkarte.html, Stand: 10.07.2020.

${ }^{4} 2017$ umbenannt in Escuela Nacional de Lenguas, Lingüística y Traducción (ENALLT).

${ }^{5}$ Jeder Bundesstaat in Mexiko hat eine Universidad Autónoma, es handelt sich um von Staatsgeldern getragene Universitäten mit eigenständiger Entscheidungsbefugnis in vielen Bereichen. Diese Universitäten bieten i.d.R. auch die schulische Oberstufe (Schuljahre 10 bis 12) an.
}

Pandaemonium, São Paulo, v. 24, n. 43, mai.-ago. 2021, p. 1-37 
Gruhn, D. H. - Publikationen der mexikanischen Germanistik im Wandel

dem auch Deutsch angeboten wird, wobei es i.d.R. Kurse für Interne, d.h. Universitätsmitarbeiter, Studenten und Schüler, und andere für externes Publikum gibt.

1966 eröffnet das Goethe-Institut eine Zweigstelle in Mexiko-Stadt (vgl. RICK 2007: 21), die bis heute existiert ${ }^{6}$. Darüber hinaus gibt es landesweit unzählige Privateinrichtungen, kleinere Privatuniversitäten, Sprachschulen und -institute, teils auch Schulketten, wie zum Beispiel Berlitz, deren Programm neben anderen Sprachen Deutsch umfasst ${ }^{7}$.

Eine geregelte Deutschlehrerausbildung gab es lange Zeit nicht im Land, es wurde bzw. wird versucht, dieses Manko mit speziellen universitären Fortbildungsveranstaltungen ${ }^{8}$ oder (Fern)Kursen des Goethe-Instituts zumindest teilweise zu beheben. Im Rahmen des seit 1955 bestehenden grundständigen Germanistikstudiengangs an der UNAM konnte sowohl nach dem früheren Studienplan von 1975, als auch nach dem neuen von 2010 ein Wahlbereich Didaktik belegt werden, der sich über vier Semester erstreckt ${ }^{9} .2005$ wurde an einer Zweigstelle der UNAM ein grundständiger Fernstudiengang (LICEL) zur akademischen Ausbildung von bereits im Berufsleben stehenden Sprachlehrern eingerichtet, bei dem Deutsch eine Option unter mehreren ist (vgl. GRÄFE 2010: 78). Als einziger germanistischer Studiengang außerhalb der UNAM existiert seit 2008 der binationale Master in Estudios interculturales de lengua, literatura y cultura alemanas (in Zusammenarbeit mit dem Herder-Institut der Universität Leipzig) an der Universidad de Guadalajara (UdeG), dessen Zielsetzung sowohl die Berufsbildung als auch die Befähigung zur Forschung umfasst $^{10}$.

\footnotetext{
${ }^{6}$ Eine weitere, früher gegründete Zweigstelle bestand zwischen 1962 und 2011 in Guadalajara, Jalisco. Deren Funktionen wurden 2017 teilweise von der Sprachschule Bezirk übernommen (vgl. RICK, 2007: 21; sowie www.milenio.com/cultura/bezirk-ya-es-centro-goethe, Stand 19.05.2020).

${ }^{7}$ Viele der Anbieter schaffen es allerdings nicht, ihre Lerner zu nachweisbaren Ergebnissen zu führen (z.B. in Form von Sprachzertifikaten). Das ist unter anderem auf die im Schnitt äußerst unbefriedigende Ausbildungssituation der als Deutschlehrer eingestellten Personen zurückzuführen.

${ }^{8}$ Herausragende Beispiele sind die einjährige Fremdsprachenlehrerausbildung am CELE der UNAM, begonnen in den 80er-Jahren (vgl. GARCIADIEGO 2007: 48) und gegenwärtig von der nun in ENALLT unbenannten Schule wieder aufgenommen, sowie das "Diplomado de profesores de alemán, de inglés y de francés" am Sprachenzentrum des Instituto Politécnico Nacional, 1995 unter der Leitung von Roland Zirpins errichtet, im Jahr 2001 aber aus administrativen Gründen wieder eingestellt (vgl. HEMING 2007: 53). Anzumerken ist, dass ein Großteil der Deutsch-Dozenten in Mexiko solche Angebote nicht nutzen konnte, unter anderem weil sie sich - was die Präsenzmodalität betrifft - fast immer auf die Hauptstadt konzentrieren.

${ }^{9} \mathrm{http}: / /$ modernas.filos.unam.mx/plan-de-estudios/, Stand: 19.05.2020.

${ }^{10}$ Vgl. www.cucsh.udg.mx/objectives_ger/master_in_deutsch_als_fremdsprache, Stand: 19.05.2020.

Pandaemonium, São Paulo, v. 24, n. 43, mai.-ago. 2021, p. 1-37
} 
Gruhn, D. H. - Publikationen der mexikanischen Germanistik im Wandel

\section{Umriss der Studie}

Ziel dieser Arbeit ist nun nicht, die Lernangebote der verschiedenen Einrichtungen weiter zu verfolgen, vielmehr liefern sie uns Eckdaten der „umfangreiche[n] Literatur zu germanistischen Themen [...], [die] in Mexiko entstanden“ ist, denn deren „bibliographische Aufarbeitung [...] bleibt ein für die weitere Entwicklung des Faches grundlegendes Forschungsdesiderat" (GRÄFE 2010: 80). Unser Beitrag hat sich vorgenommen, diese Lücke zu schmälern. Die Gesamtstudie umfasst die Aufnahme von insgesamt 1570 Titeln aus den Jahren zwischen einschließlich 1954 und 2018, welche zwecks einfacherer Weiterbehandlung in Excel-Listen übertragen wurden. Es wurde eine Zweiteilung vorgenommen in die Bereiche Germanistik im engeren Sinne (d.h. Arbeiten zu Literatur, Linguistik, Länderstudien u.a., die sich nicht explizit auf den Sprachunterricht beziehen, 1090 Titel $)^{11}$ sowie Deutsch als Fremdsprache (DaF). Darunter verstehen wir Publikationen, die sich speziell an die Zielgruppe DaF-Lehrer und -Forscher wenden, vereinzelt auch an DaF-Lerner (Lehrwerke).

Der früheste DaF-Titel ließ sich für August 1971 finden, weshalb der vorliegende zweite Teil der Studie den Zeitraum 1971-2018 umfasst. Insgesamt enthält die DaF-Liste 480 Einträge. Aufgenommen sind Titel, die in Mexiko veröffentlicht bzw. von in Mexiko dauerhaft oder vorübergehend tätigen DaF-Lehrern und -Forschern ${ }^{12}$ in anderen Ländern veröffentlicht wurden ${ }^{13}$. Für die Zeitspanne zwischen 1971 und 1994 stützen wir uns auf die Bibliografía de Estudios Germanísticos en México 1949-1994, herausgegeben von Marlene und Dietrich Rall (1994), aus der wir gemäß unserer Kriterien die Einträge aus dem DaFBereich übertragen haben, weitere Titel des Zeitraums sowie Titel der Folgejahre wurden neu aufgenommen. Begonnen hat die Suche nach neuen Titeln mit gängigen Publikationsmedien der mexikanischen Deutschlehrer (wie AMPAL-Kongressakten und der Deutschlehrerzeitschrift info-ampal). Hilfreich war, das Umfeld (Kongresse, Deutschlehrer-

\footnotetext{
${ }^{11}$ Vgl. Gruhn; Hahn (i.V.)

${ }^{12}$ Auf die gegenwärtig verbreiteten männlich-weiblichen Doppelformen möchten wir zur besseren Lesbarkeit verzichten, die weibliche Form ist jeweils mitgemeint.

${ }^{13}$ Autoren, die Mexiko nach einem vorübergehenden Aufenthalt wieder verlassen haben, sind jenseits dieser Zeitspanne nur aufgenommen, wenn sie in Mexiko bzw. in Akten von in Mexiko durchgeführten Kongressen veröffentlicht haben.

Pandaemonium, São Paulo, v. 24, n. 43, mai.-ago. 2021, p. 1-37
} 
Gruhn, D. H. - Publikationen der mexikanischen Germanistik im Wandel

verband, Autoren usw.) persönlich zu kennen. Angeschlossen hat sich eine erweiterte bibliographische Internet-Suche sowie der Besuch zweier Privatbibliotheken. Der letzte Schritt bestand in einer gezielten bibliographischen Verfolgung einzelner Autoren, die als „Vielveröffentlicher“ aufgefallen waren. Wir schätzen, die im Untersuchungszeitraum veröffentlichten DaF-Titel fast vollständig erfasst zu haben, da die DaF-Autoren in Mexiko sich in einer sehr überschaubaren Anzahl an Publikationsmedien bewegen.

Erhoben wurden vorrangig Artikel in Zeitschriften, Sammelbänden, Kongressakten (Volltexte) oder Mitteilungsblättern, Herausgebertitel und Monographien sowie Lehrwerke und deren Begleitmaterial. Weitgehend ausgenommen wurden Anthologien und Übersetzungen, Rezensionen, Berichte zu einzelnen Veranstaltungen sowie Vorstellungen von Persönlichkeiten des mexikanischen DaF-Umfelds.

Eine Mindestseitenanzahl der aufgenommenen Arbeiten wurde nicht angesetzt, insgesamt umfassen 28 Titel nur eine oder zwei Seiten und 179 Titel drei bis sechs Seiten, d.h. insgesamt 207 Titel der DaF-Liste (43,1\%) zählen nicht mehr als sechs Seiten. Das ist weniger als bei der Germanistik i.e.S. $(55,8 \%)^{14}$.

Das auf diese Weise gewonnene Material wurde verschiedenen Untersuchungskriterien unterzogen und jeweils unterschiedlich geordnet. Unser Hauptinteresse lag dabei auf allgemeinen Entwicklungstendenzen, Autoren und deren Arbeitgebern, Publikationsmitteln und Verlegern, dem Anteil einzelner Themenfelder, aber auch auf der Mitwirkung von Lektoren und Publikationssprachen. Die Ergebnisse sind nicht nur quantitativer Natur, sondern legen auch qualitative Aussagen nahe. Die Qualität der Titel als solche gehört allerdings nicht zum Untersuchungsgegenstand dieser Arbeit.

\footnotetext{
${ }^{14}$ Alle Zahlen der Studie sind auf eine Dezimastelle auf- bzw. abgerundet. Die hier vorgelegten Zahlen bilden den aktuellen Stand der Liste ab. Denkbar wäre die Umsortierung einzelner Titel in eine andere Kategorie (wenn inhaltlich mehrere Bereiche tangiert werden) oder die Hinzufügung vereinzelner, später gefundener Titel. Potenzielle Änderungen dieser Art hätten aber in jedem Fall nur geringfügige Auswirkungen auf die aktuellen Prozentangaben.

Pandaemonium, São Paulo, v. 24, n. 43, mai.-ago. 2021, p. 1-37
} 
Gruhn, D. H. - Publikationen der mexikanischen Germanistik im Wandel

\section{Anfangsjahre, Blütezeit und Rückgang}

Frühe germanistische Publikationen ab 1954 widmeten sich vorzugsweise der Literatur, der Fachbereich DaF tritt erst spät auf die Bühne und das über lange Jahre hinweg nur spärlich, wie die folgende Übersicht zeigt:

Tabelle 1: Verteilung der Publikationen im Bereich DaF nach Jahreskohorten

\begin{tabular}{|l|l|l|l|}
\hline \multicolumn{1}{|c|}{ Zeitraum } & \multicolumn{1}{|c|}{ Anzahl Jahre } & \multicolumn{1}{|c|}{ Anteil $\mathrm{n}=480$} & \multicolumn{1}{c|}{ Durchschn. Titel/Jahr } \\
\hline $1971-1989$ & 19 & $10 \%(48)$ & 2,5 \\
\hline $1990-1999$ & 10 & $28,5 \%(137)$ & 13,7 \\
\hline $2000-2009$ & 10 & $39,2 \%(188)$ & 18,8 \\
\hline $2010-2018$ & 9 & $22,3 \%(107)$ & 11,9 \\
\hline Total: 48 Jahre & Total: 48 Jahre & $100 \%=480$ & 10 \\
\hline
\end{tabular}

Quelle: eigene Darstellung.

Einige der frühen Werke haben einen „handwerklichen Charakter“: Schreibmaschinen-Layout, handschriftliche Korrekturen im gedruckten Buch und einen broschürenartigen Einband, vereinzelt wurden sie manuell zusammengeheftet. Häufig handelt es sich dabei um Unterrichtsmaterial für Lerner, das in früheren Jahrzehnten und vor der allgemeinen Zugänglichkeit des Internet noch schwer zu beschaffen war. Auch inhaltlich unterscheiden sie sich von aktuellen Herangehensweisen der Sprachdidaktik, so wirken z.B. die 20 Kleine[n] Szenen für das Klassenzimmer von Reyes Orozco (1971) - die erste DaFPublikation der Liste - aus heutiger Sicht sehr steif: Es handelt sich um festgeschriebene Dialoge zu Alltagssituationen mit detaillierten Regieanweisungen, die von den Lernern auswendig zu lernen waren.

Eine weitere Besonderheit des Zeitraums ist, dass die 48 Veröffentlichungen vor 1990 aus einem sehr engen Kreis stammen: Fast sämtliche Autoren sind von der UNAM, lediglich drei Titel haben einen „externen“ Erst- oder Zweitautor. Des Weiteren stammen 33 Titel, also 68,8\%, von DAAD-Lektoren dieses Zeitraums ${ }^{15}$. Ebenfalls 33 Titel $(68,8 \%)$ zeichnen mit dem Nachnamen Rall als Erstautor (22 mal Dietrich und 11 mal Marlene), bei zusätzlichen vierzehn Titeln sind die Ralls (gemeinsam oder einzeln) als Nebenautoren

15 DAAD-Lektoren an der UNAM: Dietrich Rall, Klaus Zimmermann, Ulrike Tallowitz; am Instituto Politécnico Nacional: Klaus Meyer-Minnemann, Roland Zirpins.

Pandaemonium, São Paulo, v. 24, n. 43, mai.-ago. 2021, p. 1-37 
Gruhn, D. H. - Publikationen der mexikanischen Germanistik im Wandel

Bild 1 und 2: Erste DaF-Publikation von 1971

Bild 3: Lesekurs von Dietrich u. Marlene Rall, UNAM 1978
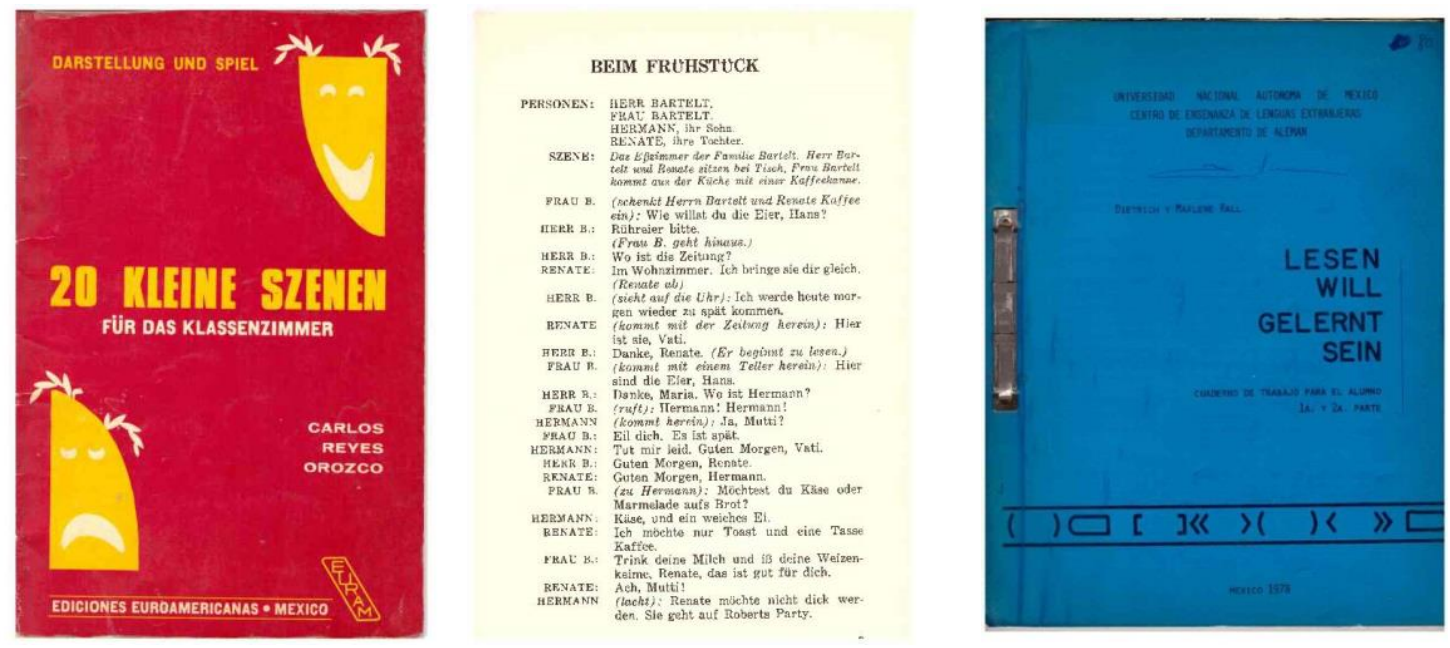

Quelle: Computerscan von Exemplaren aus meinem Privatbestand.

beteiligt. Dietrich Rall war 1969 zusammen mit seiner Ehefrau Marlene als DAAD-Lektor nach Mexiko gekommen. Das sollte für beide der Ausgangspunkt einer lebenslangen Bindung an die UNAM und eines ausgesprochen schöpferischen beruflichen Werdegangs werden, der die mexikanische Germanistik über Jahrzehnte mitprägte. Peter Pabisch und Peter Bopp (2010: 230) schreiben dazu Folgendes:

Das deutsche Gelehrtenehepaar Dieter und Marlene Rall gehörte seit den siebziger Jahren des 20. Jahrhunderts bis zum frühen Tode Marlene Ralls nach der Jahrtausendwende zu den führenden Germanisten in Lateinamerika und wirkten an der „Facultad de Filosofía y Letras“ meist in der Nachfolge Marianne Oeste de Bopp[s] ${ }^{16}$. Eine Krönung ihrer Arbeit lag in der Einberufung und Programmleitung des lateinamerikanischen Germanistenkongresses 1994 in Mexico-City, worüber sie auch einen Kongressbericht publizierten.

Doch schon vor dem Kongress des lateinamerikanischen Germanistenverbands ALEG von 1994, der eine eigene DaF-Sektion umfasste (RALL \& RALL 1996), gab es bedeutende Impulse für $\mathrm{DaF}$ in Mexiko. Im Jahre 1990 fand erstmals eine lateinamerikanische Veranstaltung statt, die sich explizit mit $\mathrm{DaF}$ beschäftigte: die „Regionalkonferenz Zielsprache Deutsch” in La Habana, Kuba. Ab diesem Jahr setzt eine umfangreichere Publikationstätigkeit im Bereich DaF ein. Anlässlich der Konferenz wurde auch ein Zusammenschluss der mexikanischen Deutschlehrer geplant, rechtskräftg gegründet

\footnotetext{
${ }^{16}$ Der Zweitautor Peter Bopp ist der Sohn von Marianne Oeste de Bopp.
}

Pandaemonium, São Paulo, v. 24, n. 43, mai.-aso. 2021, p. 1-37 
Gruhn, D. H. - Publikationen der mexikanischen Germanistik im Wandel

am 13. März 1992 als mexikanischer Deutschlehrerverein AMPAL (Asociación Mexicana de Profesores de Alemán, vgl. KRUMM et al. 2010: 1441f.). Der Verband organisierte von nun an regelmäßige Treffen in verschiedenen Bundesstaaten Mexikos und gab zwischen 1995 und 2011 insgesamt neun Tagungsakten heraus (eine davon als CD), außerdem 29 Ausgaben der Verbandszeitschrift info-ampal in den Jahren 1996 bis 2015. Seit Beginn der 90er-Jahre vervielfachten sich somit die Veröffentlichungen zu DaF-Themen und diese Hochphase sollte rund 20 Jahre lang anhalten.

Ab 2010 ist ein deutlicher Rückgang zu verzeichnen und der Abwärtstrend sticht noch stärker ins Auge, wenn man diese zeitliche Kohorte erneut unterteilt:

Tabelle 2: Verteilung der Publikationen im Bereich DaF 2010-2018

\begin{tabular}{|l|l|l|l|}
\hline \multicolumn{1}{|c|}{ Zeitraum } & \multicolumn{1}{|c|}{ Anzahl Jahre } & \multicolumn{1}{c|}{ Anteil $\mathrm{n}=107$} & \multicolumn{1}{c|}{ Durchschn. Titel/Jahr } \\
\hline $2010-2014$ & 5 & $79,4 \%(85)$ & 17 \\
\hline $2015-2018$ & 4 & $20,6 \%(22)$ & 5,5 \\
\hline Total: 9 Jahre & Total: 9 Jahre & $100 \%=107$ & 11,9 \\
\hline
\end{tabular}

Quelle: eigene Darstellung.

Zwei ALEG-Kongresse umrahmen gewissermaßen die Blützeit der mexikanischen DaF-Publikationen, denn im Jahre 2012 fand ein zweiter ALEG in Mexiko statt, diesmal in Guadalajara, Jalisco (mit Olivia Díaz als ALEG-Präsidentin und den DAAD-Lektoren Florian Gräfe und Ulrike Pless sowie der DAAD-Ortslektorin Katharina Herzig als Mitorganisatoren). Die dazugehörigen Kongressakten - ein Band ist ausschließlich der DaFDidaktik gewidmet - erschienen 2014 (HERZIG et al.).

Ab 2015 sinkt das quantitative Publikationsaufkommen auf weniger als ein Drittel des vorhergehenden Lustrums. Eine Abwanderung der vormals physischen Publikationen ins Internet kann nicht als Erklärung herangezogen werden, da Internetpublikationen im Rahmen der vorliegenden Arbeit ebenfalls erfasst wurden.

\section{Autoren und ihre Institutionen}

Es erfolgt eine Übersicht der häufigsten Institutionszugehörigkeiten der aufgenommenen Autoren (nur Erstautoren der Titel), wobei der in die DaF-Liste eingegangene Produktionsstand einzelner Autoren nicht mit ihrer Gesamtproduktion gleichgesetzt werden

Pandaemonium, São Paulo, v. 24, n. 43, mai.-ago. 2021, p. 1-37 
Gruhn, D. H. - Publikationen der mexikanischen Germanistik im Wandel

darf, manche veröffentlichen nur am Rande zu DaF-Themen, andere wiederum fallen nur für einen kurzen Zeitraum unter die Kriterien dieser Arbeit. Autoren, die an der UNAM tätig waren/sind, nehmen mit fast $60 \%$ der Einträge den ersten Platz ein. Ihr Anteil liegt somit wesentlich höher als bei der Germanistik i.e.S.. Das ist keinesfalls selbstverständlich, wenn man in Rechnung stellt, dass sich zwar der grundständige Studiengang in Letras Alemanas

Tabelle 3: Häufigste Institutionszugehörigkeiten der Autoren

\begin{tabular}{|l|l|l|l|l|}
\hline \multicolumn{1}{|c|}{ Arbeitgeber ${ }^{17}$} & Autoren & Einträge & \multicolumn{1}{|c|}{$\mathrm{n}=480$} & \multicolumn{1}{c|}{$\begin{array}{c}\text { Vergleich: Germanistik } \\
\text { i.e.S. }(\mathrm{n}=1090)\end{array}$} \\
\hline UNAM & 52 & 276 & $57,5 \%$ & $43,4 \%$ \\
\hline UdeG & 22 & 51 & $10,6 \%$ & $5,5 \%$ \\
\hline UNACH & 3 & 22 & $4,6 \%$ & unter 0,3\% \\
\hline Goethe-Institut & 8 & 16 & $3,3 \%$ & unter 0,3\% \\
\hline $\begin{array}{l}\text { Botschaftsangehörige (D, } \\
\text { AU, CH) und DAAD- } \\
\text { Zentrale }\end{array}$ & 8 & 15 & $3,1 \%$ & unter 0,3\% \\
\hline UDLAP & 3 & 15 & $3.1 \%$ & unter $0,3 \%$ \\
\hline VW-Schulen & 9 & 14 & $2.9 \%$ & unter $0,3 \%$ \\
\hline BUAP & 2 & 14 & $2,9 \%$ & $1,5 \%$ \\
\hline $\begin{array}{l}\text { ColAl (Mex, Pue, Guad.) } \\
\text { u.ZfA }\end{array}$ & 7 & 13 & $2,7 \%$ & unter $0,3 \%$ \\
\hline UAM & 3 & 10 & $2,1 \%$ & $6,7 \%$ \\
\hline Sonstige & 21 & 34 & $7,1 \%$ & \\
\hline
\end{tabular}

Quelle: eigene Darstellung.

an der UNAM befindet und an dem dortigen Sprachenzentrum (CELE bzw. ENALLT) Forscherstellen existieren, es aber landesweit Deutschlehrer an sehr vielen Institutionen gibt. Auf den ersten Blick wäre also eine viel breitere Streuung der institutionellen Zugehörigkeit der DaF-Autoren zu erwarten gewesen.

Auf Platz zwei liegt mit knapp 11\%, die UdeG, deren binationaler Master erst 2008 angelaufen ist (wobei nicht alle aufgenommenen Titel der UdeG mit diesem Master in Verbindung stehen). Alle übrigen Institutionen und Organismen befinden sich unterhalb der 5\%-Marke. Besonders zu erwähnen ist die UNACH in Chiapas, denn fast alle Einträge (19)

\footnotetext{
${ }^{17}$ UNAM: Universidad Nacional Autónoma de México, aufgenommen sind alle Zweigstellen der UNAM, also sowohl Facultad de Filosofía y Letras, CELE bzw. ENALLT sowie FES-Acatlán u.a.; UdeG: Universidad de Guadalajara; UNACH: Universidad Autónoma de Chiapas; Goethe-Institut in Mexiko-Stadt; VW-Schulen: Centro de Idiomas Volkswagen mit vier Zweigstellen; UDLAP: Universidad de las Américas Puebla; ColAl: Colegio Alemán (Humboldt-Schulen in Mexiko-Stadt und Puebla); BUAP: Benemérita Universidad Autónoma de Puebla; UAM: Universidad Autónoma Metropolitana.
}

Pandaemonium, São Paulo, v. 24, n. 43, mai.-ago. 2021, p. 1-37 
Gruhn, D. H. - Publikationen der mexikanischen Germanistik im Wandel

dieser Universität stammen von einem einzigen Autor: Martin Dettmer, äußerst aktives Mitglied des Deutschlehrerverbands seit der Gründerzeit ${ }^{18}$.

Man kann es auch aus anderer Perspektive betrachten: 72,7\% aller Einträge der DaFListe stammen von Autoren, die an nur drei öffentlichen Universitäten beschäftigt waren/sind.

Beachtlich ist, dass bei einer Eingrenzung der Autoren auf diejenigen, die mindestens 5 Einträge in der Germanistikliste haben, nur insgesamt 24 Autoren für 302 aller Einträge des Gesamtzeitraums - fast ein halbes Jahrhundert (1971-2018) - zeichnen, das entspricht 62,9\%. Nachfolgend die entsprechende Übersicht:

Tabelle 4: „Vielveröffentlicher“ (ab 5 Einträge)

\begin{tabular}{|l|l|l|l|c|}
\hline \multicolumn{5}{|c|}{ Arbesamtliste DaF $(\mathrm{n}=480)$} \\
\hline \multicolumn{1}{|c|}{ Name } & \multicolumn{1}{|c|}{ Zeitraum } & \multicolumn{1}{c|}{ Stand 2020 } & Einträge \\
\hline Rall, Dietrich & UNAM, DAAD-L & $1975-2014$ & pensioniert & 45 \\
\hline Böhm ${ }^{19}$, Siegfried & UNAM & $1996-2015$ & aktiv & 41 \\
\hline Rall, Marlene & UNAM & $1975-2002$ & verstorben & 40 \\
\hline Dettmer, Martin & UNACH, Chis., DAAD-OL & $1992-2011$ & verstorben & 19 \\
\hline Bauer, Ulrich & UNAM, DAAD-L & $2003-2008$ & Mex. verl. & 12 \\
\hline Mager, Elisabeth & UNAM & $1996-2012$ & aktiv & 13 \\
\hline Gruhn, Heike & BUAP, DAAD-OL & $1995-2018$ & aktiv & 13 \\
\hline Herzig, Katharina & UdeG, DAAD-OL & $2004-2014$ & aktiv & 11 \\
\hline Fandrych, Christian & UNAM, DAAD-L & $1995-2001 / 2017$ & Mex. verl. & 11 \\
\hline Heckel, Ilse & UNAM & $1971-2000$ & verstorben & 10 \\
\hline Terborg, Roland & UNAM & $1995-2005$ & aktiv & 9 \\
\hline Elorduy, Ma. Esther & UNAM & $1989-1998$ & verstorben & 7 \\
\hline Ecke, Peter & UDLAP, Univ. of & $1998-2011$ & Mex. verl. & 7 \\
\hline Hausstein, Alexandra & EU-Projekt Mex.-Stadt & $2003-2008$ & Mex. verl. & 7 \\
\hline Sperr, Ulrike & UDLAP & $2002-2013$ & aktiv & 7 \\
\hline Hirschfeld, Diana & UNAM & $2001-2014$ & aktiv & 6 \\
\hline $\begin{array}{l}\text { Rivera Ochoa, Ma. } \\
\text { Clotilde }\end{array}$ & UAM & $1993-2000$ & pensioniert & 6 \\
\hline $\begin{array}{l}\text { Au, Alexander } \\
\text { García Llampallas, } \\
\text { Claudia }\end{array}$ & UdeG, DAAD-L (DAAD- & $2003-2005$ & Mex. verl. & 5 \\
\hline Garciadiego, Rubén & UNAM & $1996-2010$ & aktiv & 5 \\
\hline Lauterbach, Stefan & UNAM-DAAD-L & $2000-2005$ & Mex. verl. & 5 \\
\hline Tallowitz, Ulrike & UNAM-DAAD-L & $1985-1998$ & Mex. verl. & 5 \\
\hline Willkop, Eva Maria & UNAM-DAAD-L & $1991-2002$ & Mex. verl. & 5 \\
\hline
\end{tabular}

\footnotetext{
${ }^{18}$ Martin Dettmer ist im Dezember 2014 unerwartet verstorben.

${ }^{19}$ Eigentlich Böhm, in spanischsprachigen Publikationen aber i.d.R. Boehm geschrieben.
}

Pandaemonium, São Paulo, v. 24, n. 43, mai.-ago. 2021, p. 1-37 
Gruhn, D. H. - Publikationen der mexikanischen Germanistik im Wandel

\begin{tabular}{|c|l|l|c|c|}
\hline Wossidlo, Eckart & $\begin{array}{l}\text { Deutsche Schule, Mex.- } \\
\text { Stadt }\end{array}$ & $1996-2004$ & pensioniert & 5 \\
\hline 24 & & & & $302=$ \\
& & & $62,9 \%$ \\
\hline
\end{tabular}

Quelle: eigene Darstellung.

Dieser Wert ist fast identisch mit der Liste der Germanistik i.e.S., wo 23 Personen (mit mindestens 7 Einträgen) hinter 63,9\% aller Einträge stehen, vier Autoren erscheinen in beiden Listen unter den ,Vielveröffentlichern“. Aufgenommen sind in Tabelle 4 nur Erstautoren, dieselben „Vielveröffentlicher“ treten aber darüber hinaus auch häufig als Zweit- oder Drittautoren auf. Bei 63\% davon handelt es sich um Dozenten der UNAM, bei den übrigen sind die Zugehörigkeiten gestreut. 42\% waren/sind DAAD-Lektoren oder Ortslektoren (bei der Germanistik i.e.S: nur rund 25\%). Die Erstsprache von 83\% ist Deutsch, $67 \%$ haben bzw. hatten ihren Lebensmittelpunkt in Mexiko, die übrigen haben Mexiko nach Ablauf ihres Lektorats bzw. anderweitiger Funktionen wieder verlassen.

Alles in Allem ergibt sich kein vielversprechendes Panorama: Von den 24 „Vielveröffentlichern“ sind vier bereits verstorben, haben acht das Land (teilweise schon vor Langem) definitiv verlassen und sind drei pensioniert. Von den neun „Vielveröffentlichern“, die in Mexiko noch beruflich aktiv sind, werden die meisten in absehbarer Zeit in den Ruhestand gehen; einige schreiben kaum noch zu DaF, z.B. weil sie in andere Fachbereiche abgewandert sind. Das bedeutet: nur von den wenigsten sind auch in Zukunft noch Veröffentlichungen zu erwarten. Eine ebenso produktive Nachfolgergeneration zeichnet sich nicht $\mathrm{ab}$.

Die vorstehende Tabelle liefert auch Anhaltspunkte, die außerordentliche Dominanz der UNAM bei den DaF-Publikationen zu erklären. Waren doch einige dieser „Vielveröffentlicher“ der UNAM Gründungsmitglieder von AMPAL und/oder haben regelmäßig in den Schriften des Verbands veröffentlicht. Über Jahre hinweg findet sich dort ein fester Autorenstamm, der Artikel, Berichte und andere Textsorten publiziert ${ }^{2021}$. Die

\footnotetext{
${ }^{20}$ Dazu gehören u.a. Dietrich und Marlene Rall, Diana Hirschfeld, Rubén Garciadiego, Roland Terborg, Ilse Heckel, Siegfried Böhm, Elisabeth Mager, Esther Elorduy, Claudia García Llampallas, darüber hinaus auch DAAD-Lektoren, die sich vorübergehend im Land aufhielten, wie Ulrich Bauer, Stefan Lauterbach und Christian Fandrych.

${ }^{21}$ Nicht alle Titel der AMPAL-Publikationen finden sich in der DaF-Liste, einige wurden auch der Germanistik i.e.S. zugeordnet. Viele Autoren finden sich in beiden Listen.
}

Pandaemonium, São Paulo, v. 24, n. 43, mai.-ago. 2021, p. 1-37 
Gruhn, D. H. - Publikationen der mexikanischen Germanistik im Wandel

UNAM ist also nicht nur die Wiege der universitären Germanistik in Mexiko, sondern mittels des Deutschlehrerverbands AMPAL - auch der DaF-Publikationen im Land.

Was die übrigen Autoren betrifft, so handelt es sich dabei nicht zwangsweise um Personen, die wenig veröffentlichen, sondern lediglich um solche, die wenige Titel nach den Kriterien der hier zusammengestellten DaF-Liste haben. Einige davon kommen auf eine Vielzahl an Einträgen, wenn man ihre Titel in dieser Liste mit denen der Germanistik i.e.S. zusammenzählt ${ }^{22}$.

Die Mehrzahl der übrigen 178 Einträge stammt allerdings von Autoren, die nicht eng an den Wissenschaftsbetrieb angebunden sind. Dabei handelt es sich zum Teil um Vertreter von offiziellen Organismen oder Institutionen (Botschaften, Goethe-Institut, Zentralstelle für Auslandsschulwesen), die häufig mit Lagebeschreibungen (Berichten) zu den DaFPublikationen beisteuerten, aber auch um Deutschlehrer, die nur gelegentlich zur Feder griffen bzw. greifen. Diese Personengruppe veröffentlichte vor allem in AMPALPublikationsmedien, was sich anhand der Zahlen belegen lässt:

Tabelle 5: Autoren und Einträge in AMPAL-Publikationsmedien

\begin{tabular}{|l|l|l|l|}
\hline & \multicolumn{1}{|c|}{ „Vielveröffentlicher“ } & Übrige Autoren & Gesamt \\
\hline Autoren & 24 & 114 & 138 \\
\hline Einträge & $302(\mathrm{n}=302)$ & $178(\mathrm{n}=178)$ & 480 \\
\hline $\begin{array}{l}\text { Davon in AMPAL- } \\
\begin{array}{l}\text { Publikationen (Zeitschrift, } \\
\text { Kongressakten, Sammelband) }\end{array}\end{array}$ & $118(39,1 \%)$ & $103(=57,9 \%)$ & 221 \\
\hline Nicht-AMPAL-Publikationen & $184(60,9 \%)$ & & 259 \\
\hline
\end{tabular}

Quelle: eigene Darstellung.

Bei den „Vielveröffentlichern“ lässt sich eine wesentlich breitere Streuung der Publikations-medien feststellen, sie publizierten mehr als 60\% ihrer Titel jenseits von AMPAL. Von den insgesamt 24 Autoren dieser Rubrik haben alle mindestens einen Eintrag in einem AMPAL-Medium, 23 (96\%) auch Einträge in anderen Medien, 16 davon z.B. mindestens einen Eintrag in einem ALEG-Tagungsband oder in der DaF-Brücke und 18 (75\%) haben darüber hinaus Einträge jenseits der vorgenannten Medien, d.h. in anderen mexikanischen oder ausländischen Fachzeitschriften, Sammelbanden, Kongressakten oder Monographien.

\footnotetext{
${ }^{22}$ Z.B. Christine Hüttinger, Sabine Pfleger, Olivia Díaz Pérez, Klaus Zimmermann u.a.

Pandaemonium, São Paulo, v. 24, n. 43, mai.-ago. 2021, p. 1-37
} 
Gruhn, D. H. - Publikationen der mexikanischen Germanistik im Wandel

Bei den übrigen Autoren fällt der Anteil der Titel in Publikationsmedien jenseits von AMPAL auf 42,1\%. Oder aus anderer Perspektive betrachtet: nur rund ein Drittel der Autoren haben auch AMPAL-fremd veröffentlicht, hier vor allem in ALEG-Kongressakten.

Deutlich wird, dass die Einstellung der AMPAL-Publikationsmedien sowie der Abgang der meisten „Vielveröffentlicher“ unvermeidlich zu einem starken zahlenmäßigen Zusammenschrumpfen der DaF-Publikationen führen musste.

\section{Zur Rolle der (Orts-)Lektoren}

Wie schon mehrfach betont wurde, waren (Orts-)Lektoren wesentlich an den mexikanischen DaF-Publikationen beteiligt. Zu unterscheiden sind Lektoren des Deutschen Akademischen Austauschdienstes (DAAD), Lektoren des Österreichischen Austauschdienstes (OeAD) und DAAD-Ortslektoren, die an einer mexikanischen Universität arbeiten, aber nicht an bestimmte (Lektoren-)Standorte gebunden sind. Letztere haben keine speziellen Pflichten, vielmehr erhalten sie Unterstützung in Form von Materialien, Reisekostenzuschüssen u.a. Für die vorliegende Arbeit klassifizieren wir alle Personen als (Orts-)Lektoren, die innerhalb des untersuchten Gesamtzeitraums, d.h. zwischen 1955 und 2018, einmal als solche fungierten, und zwar durchgehend, in der gesamten Liste, nicht nur für die Jahre, die sie die entsprechende Ernennung innehatten. Das, weil wir davon ausgehen, dass ein solches Lektorat langfristige Kontakte schafft und nachhaltig Wirkungen zeitigt.

Da weder von deutscher noch österreichischer Seite ein vollständiges Lektorenregister für Mexiko zur Verfügung stand, stellten wir die entsprechenden Listen aus verschiedenen Quellen zusammen. Insgesamt konnten wir für den Zeitraum zwischen 1965 und 201845 DAAD-Lektoren an fünf mexikanischen Standorten erfassen: UNAM-CU, UNAM-Acatlán sowie CENLEX/IPN in Mexiko-Stadt, UANL in Monterrey und UdeG in Guadalajara; darüber hinaus vier DAAD-Ortslektoren (1989-2018) sowie 14 OeADLektoren (1983-2018) an denselben Standorten wie die ersteren (außer CENLEX/IPN) ${ }^{23}$.

\footnotetext{
${ }^{23}$ Martin Rauchbauer (2007: 8) setzt das Programm der Österreich-Lektoren 1994 an, wir fanden allerdings eine Österreich-Lektorin für 1983-1986 (UNAM-Acatlán), evtl. eine Vorstufe des Programms.

Pandaemonium, São Paulo, v. 24, n. 43, mai.-ago. 2021, p. 1-37
} 
Gruhn, D. H. - Publikationen der mexikanischen Germanistik im Wandel

Von diesen insgesamt 63 Personen haben 29 (46\%) Arbeiten veröffentlicht, die in die DaFListe eingegangen $\operatorname{sind}^{24}$ :

Tabelle 6: Lektoren und Ortslektoren

\begin{tabular}{|l|l|l|l|}
\hline & \multicolumn{1}{|c|}{ DaF-Liste (Personen) } & \multicolumn{1}{c|}{ Einträge (1971-2018) } & \multicolumn{1}{c|}{$\% \mathrm{n}=480$} \\
\hline DAAD-Lektoren & 21 & 140 & $29,2 \%$ \\
\hline DAAD-Ortslektoren & 3 & 44 & $9,2 \%$ \\
\hline OeAD-Lektoren & 5 & 9 & $1,9 \%$ \\
\hline Gesamt & 29 & 193 & $40,2 \%$ \\
\hline
\end{tabular}

Quelle: eigene Darstellung.

Die Investition in Lektoren und Ortslektoren hat sich bezüglich der Publikationen als äußerst fruchtbar erwiesen. Im Vergleich zur Germanistik i.e.S. lässt sich sagen, dass die Lektoren insgesamt mehr im DaF-Bereich veröffentlichten (193/480 gegen 174/1090) und ihr prozentualer Anteil deshalb dort wesentlich höher ausfällt (40,2\% gegen 16\%).

Des Weiteren ist festzuhalten, dass die Lektoren-Einträge kohortenmäßig rückläufig sind. Lag bis 1989 der Anteil der DAAD-Lektoren (andere waren damals noch nicht beteiligt), wie oben schon angemerkt, bei $68,8 \%$, so sinkt der Lektoren-Anteil (nun aller Lektorentypen) auf 34,8\% für den Zeitraum zwischen 1990 und 2009 (bei insgesamt wesentlich mehr Veröffentlichungen). Ab 2010 beträgt er dann nur noch knapp 29\%.

Eine besonders intensive Mitwirkung haben sie bei Herausgeberschriften (76\% der aufgenommenen Herausgebertitel entstanden unter Mitwirkung von (Orts-)Lektoren), sowie allgemein bei Sammelbänden (56,7\%). Was die inhaltlichen Bereiche betrifft, so sind (Orts)Lektoren überdurchschnittlich bei Berichten (56,3\%) und Arbeiten rund um Lehrwerke $(53,6 \%)$ beteiligt.

Bis vor wenigen Jahren gehörte die Förderung der germanistischen Studien und wissenschaftlichen Entwicklung im Ausland ausdrücklich zur Zielsetzung des DAAD (vgl. SPITTA 2007: 19), was auch die Finanzierung verlegerischer Projekte beinhaltete.

\footnotetext{
${ }^{24}$ Wobei der langjährige DAAD-Außenstellenleiter in Mexiko-Stadt Arnold Spitta auch mit eingerechnet wird, ebenso sein Nachfolger Alexander Au, der aber als ehemaliger DAAD-Lektor ohnehin in der Liste erfasst ist.

Pandaemonium, São Paulo, v. 24, n. 43, mai.-ago. 2021, p. 1-37
} 


\section{Publikationsmedien und Verleger}

\subsection{Publikationsmedien}

Zunächst eine kurze Übersicht, auf welche Medien sich die DaF-Publikationen verteilen:

Tabelle 7: DaF-Publikationsmedien

\begin{tabular}{|l|l|l|l|}
\hline \multicolumn{1}{|c|}{ Publikationsmedien } & \multicolumn{1}{|c|}{ Einträge } & \multicolumn{1}{|c|}{$\mathrm{n}=480$} & \multicolumn{1}{c|}{$\begin{array}{c}\text { Davon von AMPAL hg. und verlegt; } \\
\mathrm{n}=480\end{array}$} \\
\hline Kongressakten & 206 & $42,9 \%$ & 131 \\
\hline Zeitschriftenartikel & 157 & $32,7 \%$ & 70 \\
\hline Sammelband & 93 & $19,4 \%$ & $20^{25}$ \\
\hline Sonstige & 24 & $5 \%$ & 0 \\
\hline & 480 & $100 \%$ & $221=46 \%$ \\
\hline
\end{tabular}

Quelle: eigene Darstellung.

\section{Kongressakten}

Was die Publikationsmedien betrifft, so stehen die Kongressakten ${ }^{26}$ mit knapp 43\%, mit Abstand an erster Stelle, dreizehn davon wurden als CD herausgegeben. 63,6\% aller Akten stammen von AMPAL-Kongressen (I Encuentro AMPAL 1993 - $\mathrm{X}^{\circ}$ Encuentro AMPAL/Jornadas Dach 2011). Darüber hinaus lassen sich 31 Beiträge in den Akten anderer nationaler Kongresse finden, die meisten davon (22) wurden von der UNAM editiert.

An zweiter Stelle stehen Beiträge in ALEG-Kongressen (34), insbesondere dann, wenn diese in Mexiko stattgefunden haben, was im Jahr 1994 (ALEG VIII in Mexiko-Stadt) und 2012 (ALEG XIV in Guadalajara) der Fall war. Wobei nur Band 4 (HERZIG et al. 2014) der DaF-Liste zugeordnet wurde ${ }^{27}$. Insgesamt haben die Vertreter von DaF in Mexiko wenig Präsenz bei Kongressen in anderen lateinamerikanischen Ländern gezeigt, am ehesten noch auf Kuba - offensichtlich das beliebteste Auslandsziel der mexikanischen DaF-Dozenten. Nach 2014 zeichnet sich ein weitgehendes Verschwinden von Beiträgen in diesem vormals

\footnotetext{
${ }^{25}$ Es handelt sich nicht um 20 Sammelbände, sondern um zwanzig Einträge (Herausgeberschaft oder Artikel) in Verbindung mit mehreren Sammelbänden.

26 Kongressakten: Publikation der Volltexte, die infolge von Kongressen, Tagungen und ähnlichen Veranstaltungen entstanden sind. Als Synonym wird in diesem Artikel auch Tagungsakten verwendet.

${ }^{27}$ Darüber hinaus ein Text aus Band 3, der sich mit Übersetzen im Fremdsprachenunterricht beschäftigt. Andere Titel aus den Bänden 1 bis 3 dieses Kongresses finden sich in der Liste zur Germanistik i.e.S.
}

Pandaemonium, São Paulo, v. 24, n. 43, mai.-ago. 2021, p. 1-37 
Gruhn, D. H. - Publikationen der mexikanischen Germanistik im Wandel

beliebtesten Publikationsmedium ab, und zwar sowohl auf nationaler wie lateinamerikanischer Ebene ${ }^{28}$.

Fünf der aufgenommenen Titel in Kongressakten sind infolge von Vorträgen bei internationalen Deutschlehrertagungen in Europa entstanden und auch dort verlegt worden.

\section{Zeitschriftenartikel}

Die Zeitschriftenartikel liegen mit 32,7\% an zweiter Stelle. Autoren der UNAM sind mit 47,8\% der Titel vertreten, Autoren der UdeG mit 15,3\%, beide Institutionen decken für sich allein also $63 \%$ der Zeitschrifteneinträge ab. 42\% stammen von (Orts-)Lektoren. Besonders häufig haben Dietrich und Marlene Rall in Zeitschriften veröffentlicht, zusammen stellen sie 20,4\% aller Einträge, gefolgt von Siegfried Böhm mit 5\%.

Das Publikationsvolumen in Zeitschriften vor 1990 lag im Schnitt bei 1,1 Titel/Jahr, im Zeitraum von 1990 bis 1999 bei 4,6 T./Jahr, im Jahrzehnt 2000-2009 stieg es auf 6,3 T./Jahr an - somit die in quantitaver Hinsicht ertragreichste Periode -, ab 2010 sind es dann nur noch 3,4 T./Jahr, d.h. es wurde wieder deutlich weniger in Zeitschriften publiziert. Betrachtet man die Jahre 2015-2018 getrennt, fällt die Zahl mit 1,5 T./Jahr praktisch auf den Stand vor 1990 zurück.

Die Zeitschriftenartikel im Bereich DaF tendieren dazu, kurz zu sein, 22 haben nicht mehr als zwei Seiten, 87 zwischen drei und sechs Seiten, das sind zusammen 69,4\%. Nachfolgend eine Tabelle, aus der hervorgeht, in welchen Zeitschriften vorrangig veröffentlicht wurde:

Tabelle 8: Häufigste Zeitschriften

\begin{tabular}{|l|l|l|}
\hline \multicolumn{1}{|c|}{ Zeitschrift } & \multicolumn{1}{c|}{ Verleger } & \multicolumn{1}{c|}{ Gesamteinträge, $\mathrm{n}=157$} \\
\hline info-ampal & AMPAL & 70 \\
\hline Estudios de Lingüística Aplicada $($ ELA $)$ & UNAM & 23 \\
\hline DaF-Brücke & DeLiLa $^{29}$ & 15 \\
\hline & & $\mathbf{1 0 8}=\mathbf{6 8 , 8 \%}$ \\
\hline
\end{tabular}

Quelle: eigene Darstellung.

\footnotetext{
${ }^{28}$ Die Akten zweier internationaler Kongresse an der UNAM finden sich unter den Sammelbänden.

${ }^{29}$ Projekt Deutschlehrer in Lateinamerika.

Pandaemonium, São Paulo, v. 24, n. 43, mai.-ago. 2021, p. 1-37
} 
Gruhn, D. H. - Publikationen der mexikanischen Germanistik im Wandel

Drei Zeitschriften zusammen decken also knapp 69\% aller Einträge der Rubrik ab. Bei den übrigen rund $30 \%$ ist keine besondere Häufung zu beobachten. 24 Einträge beziehen sich auf Fachzeitschriften, die in deutschsprachigen Ländern bzw. von einem Goethe-Institut verlegt wurden, hier erscheint Fremdsprache Deutsch am häufigsten (8 Einträge), weitere sind Zielsprache Deutsch, Deutsch als Fremdsprache, u.a. Die 2013 gegründete onlineZeitschrift Verbum et Lingua der UdeG, in der explizit auch auf Deutsch veröffentlicht werden kann, verzeichnet nur drei DaF-Einträge.

Die Zeitschrift des Mexikanischen Deutschlehrerverbands, info-ampal (1996-2015) hatte $\mathrm{zu}$ keiner Zeit ein wissenschaftliches Profil. Neben Artikeln, die i.d.R. wissenschaftliche Erkenntnisse vereinfacht darstellten, überwogen die Textsorten didaktische Hilfestellungen und Erfahrungsberichte, Beschreibung von neuen Unterrichtsmaterialien oder -medien, Berichte (von Veranstaltungen, Gruppenreisen, Forschungsaufenthalten u.a.), Interviews bzw. Vorstellung von Persönlichkeiten, Institutionen oder Sprachabteilungen (z.B. der Sprachleitung des Goethe-Instituts, Kulturbeauftragte an den Botschaften usw.), Gratulationen, Verabschiedungen (Ruhestand, Weggang), Nachrufe, Ankündigungen, darüber hinaus auch Rezensionen. In der diesem Artikel zugrunde liegenden DaF-Liste haben wir ausschließlich die als Artikel gekennzeichneten Texte der Zeitschrift übernommen, es waren im Schnitt zwischen 2 und 4 pro Ausgabe. Ebenso wie die AMPAL-Kongressakten wurde info-ampal im Rahmen der regelmäßig stattfindenden Präsenzveranstaltung kostenlos verteilt, teilweise auch eigens verschickt, und erfreute sich großer Beliebtheit unter den Mitgliedern des Vereins.

Ab Anfang des 21. Jahrhunderts besteht innerhalb der mexikanischen Universitäten ein starker Druck, in indizierten Zeitschriften zu veröffentlichen (vgl. VASEN; LUJANO 2017). Dabei handelt es sich um periodisch erscheinende Fachzeitschriften, die fest umrissenene Standards zu erfüllen haben, um in bestimmte länderübergreifende Kataloge ${ }^{30}$ aufgenommen $\mathrm{zu}$ werden. Artikel, die in solchen Zeitschriften veröffentlicht werden, genügen strengen formalen Kriterien und wurden vor ihrer Annahme einem Doppelblindgutachten unterzogen. Um das Jahr 2005 sind die meisten universitären Zeitschriften in Mexiko und Lateinamerika

\footnotetext{
${ }^{30}$ Dieser Arbeit liegt die Aufnahme bei Latindex zu Grunde: www.latindex.org/latindex/inicio [Stand: März 2020].

Pandaemonium, São Paulo, v. 24, n. 43, mai.-ago. 2021, p. 1-37
} 
Gruhn, D. H. - Publikationen der mexikanischen Germanistik im Wandel

zu so einer revista indexada aufgestiegen, Estudios de Lingüística Aplicada (UNAM) im November 2005, Verbum et Lingua (UdeG) ab 2015. Zeitschriften, die diesen Katalogstandards nicht genügen, sind im akademischen Umfeld praktisch wertlos und deshalb größtenteils eingestellt worden. Insgesamt sind nur 3 Titel der DaF-Liste in einer Zeitschrift herausgekommen, als diese bereits bei Latindex indiziert war $^{31}$.

Autoren, die auf universitäre Stimuli angewiesen sind, können es sich gegenwärtig kaum noch leisten, in nicht indizierten Zeitschriften zu schreiben. Wohin die Autorenschaft der 2015 zum letzten Mal erschienenen Verbandszeitschrift info-ampal abgewandert ist, lässt sich nicht feststellen. Fachzeitschriften, die in den deutschsprachigen Ländern verlegt werden, verzeichnen jedenfalls keinen Zugang mexikanischer DaF-Autoren (insgesamt nur 7 Einträge ab 2005). Vielmehr muss vermutet werden, dass vor allem der Personenkreis, der vormals nur gelegentliche Beiträge leistete, seit diesem Datum nicht in anderen Zeitschriften $^{32}$, sondern überhaupt nicht mehr (in diesem Medium) veröffentlicht.

\section{Sammelbände}

Was die Sammelbände betrifft, so stehen diese mit 93 Einträgen $(19,4 \%)$ an dritter Stelle. Aufgenommen wurde jeweils die Herausgeberschaft (insgesamt 8 Herausgebertitel), sowie einzelne Beiträge in diesen sowie weiteren Sammelbänden, deren Herausgeberschaft nicht der mexikanischen Germanistik zugerechnet wird.

Bei den deutschen Verlagen erzielt der Universitätsverlag Göttingen die meisten Einträge (14), gefolgt von Iudicium München, da wir Artikel im Jahrbuch Deutsch als Fremdsprache (neun von Dietrich und/oder Marlene Rall sowie einer von Christian Fandrych) unter die Rubrik Sammelband eingeordnet haben. Zwei der beim Universitätsverlag Göttingen in der Reihe Materialien Deutsch als Fremdsprache erschienenen Bände sind infolge von internationalen Kongressen entstanden, die jeweils 2013 und 2016 an der UNAM stattfanden (BERNSTEIN; LERCHNER 2014: Ästhetisches Lernen im DaF-/DaZ-Unterricht, HAASE; HöLlER 2017: Kulturelles Lernen im DaF/DaZ-

\footnotetext{
${ }^{31}$ Einige Beiträge bei Verbum et Lingua sind in der Liste der Germanistik i.e.S. enthalten.

${ }^{32}$ In Mexiko gibt es gegenwärtig keine germanistische bzw. DaF-Fachzeitschrift. Lateinamerikanische Fachzeitschriften sind die ungefähr alle drei Jahre erscheinende überregionale DaF-Brücke und Pandaemonium Germanicum der Universidade de São Paulo.

Pandaemonium, São Paulo, v. 24, n. 43, mai.-ago. 2021, p. 1-37
} 
Gruhn, D. H. - Publikationen der mexikanischen Germanistik im Wandel

Unterricht). Unter den Herausgebern sind zwei DAAD-Lektoren und eine OeAD-Lektorin. Wir haben sie den Sammelbänden zugeordnet, weil sich in dieser Kategorie schon weitere, im selben Verlag veröffentlichte Titel befinden ${ }^{33}$. Es handelt sich um die jüngsten Sammelbände, zusammen mit einem weiteren, 2017 von der UdeG verlegt (DíAZ PÉREZ et al. $2017 \mathrm{a})^{34}$.

Eine Sonderstellung nimmt El idioma alemán en México. Medio de enlace, oportunidades y prestigio (AMPAL et al. 2007) ein. Hierbei handelt es sich um das einzige von AMPAL jenseits der regulären Kongressakten herausgegebene Buch, und zwar in Kooperation mit dem DAAD und der Universidad Autónoma de Baja California. Hervorgegangen ist es aus einem Fachsymposium im Jahre 2006 in Tijuana, Baja California, das sich der Lagebeschreibung von Deutsch als Fremdsprache widmete; der Band enthält also auschließlich DaF-Lage-Berichte. Er wurde ebenfalls als Sammelband klassifiziert.

Wie oben schon festgehalten, haben (Orts-)Lektoren überdurchschnittlich viele Einträge in der Rubrik Sammelband (56,7\%), allen voran DAAD-Lektoren. Drei Autoren vereinen für sich allein 43,3\% der Titel (Dietrich und Marlene Rall sowie Siegfried Böhm), bei den übrigen lässt sich keine Konzentration erkennen. Inhaltlich sticht die Textsorte „Bericht“ mit 41,1\% der Einträge heraus, die anderen Titel widmen sich unterschiedlichen Themenfeldern.

\section{Sonstiges}

Bei den restlichen 24 Einträgen (Sonstiges, 5\%) dominieren Lehrwerke und deren Begleitmaterialien sowie Grammatikzusammenfassungen (zusammen 15 Titel), allen voran Sprachbrücke II und I (MEBUS et al. 1989, 1994), aber auch Lesekurse (RALL 1978, TALlowitz 1985, BOEHM 2008/2013), ein Lehrerhandbuch zur Dramapädagogik (BOEHM 2013) sowie drei Mimeos. Die DaF-Gesamtliste enthält lediglich drei Monographien, zwei von Ilse Heckel $(1984 ; 1992)$ jeweils zu Theater und Lautlehre des Deutschen sowie ein weiteres Werk zur Lautlehre (MADRID \& ZIRPINS 1988).

\footnotetext{
${ }^{33}$ Ähnlich verhält es sich mit dem beim Stauffenburg-Verlag verlegten Band 4 von ALEG XIV, diesen haben wir jedoch bei den Kongressakten belassen, da er mit den Akten anderer ALEG-Kongresse eine Einheit bildet. ${ }^{34}$ Ein weiterer, im selben Jahr in Deutschland erschienener Sammelband unter Mitherausgeberschaft von Olivia Díaz (2017b) wurde in der Liste zur Germanistik i.e.S. aufgenommen.

Pandaemonium, São Paulo, v. 24, n. 43, mai.-ago. 2021, p. 1-37
} 
Gruhn, D. H. - Publikationen der mexikanischen Germanistik im Wandel

Autorenzusammensetzung und ,, versteckte Publikationen“

Anzumerken ist, dass die zwei oben genanten jüngsten Herausgaben (2014 und 2017), die infolge von an der UNAM durchgeführten internationalen Kongressen entstanden sind, nur bei oberflächlicher Betrachtung ein Vitalitätszeichen der mexikanischen DaFPublikationslandschaft der letzten Erhebungsjahre senden. Zwar ist es im Rahmen von internationalen Veranstaltungen üblich, für die dazugehörgen Tagungsakten auch internationale Autoren zu gewinnen. Ebenso üblich ist allerdings, dass eine bedeutende Veranstaltung des Fachbereichs die vorhandenen Kräfte vor Ort mobilisiert, nicht nur als Organisatoren und Publikum, sondern eben auch als Autoren. In den beiden genannten Sammelbänden sind es allerdings nur jeweils $20 \%$ und 22\% der Autoren, die unter die hiesigen Kriterien der mexikanischen Germanistik fallen (im Übrigen haben die meisten davon Mexiko inzwischen wieder verlassen). Das bedeutet, dass ein fester, in Mexiko ansässiger Autorenstamm kaum noch aktiviert werden konnte. Zum Vergleich: In der Sektion 3 (Alemán como lengua extranjera y lingüística aplicada) der Actas del VIII ALEG in Mexiko-Stadt (RALL \& RALL 1996), können 40,9\% der Autoren der mexikanischen Germanistik zugerechnet werden (die Mehrheit davon ortsansässig).

Ein weiteres, gegenwärtig immer häufiger auftretendes Phänomen sind ,,versteckte Publikationen“. Publikationszwang, Krise des Verlagswesens und Mittelkürzungen führen zu Veröffentlichungen in geringer Auflagenzahl bzw. (online-)Veröffentlichungen, bei denen die (auch universitären) Verlage sich nicht um eine wirksame Verbreitung kümmern. Das Ergebnis sind Werke, die zwar existieren, i.d.R. auch eine ISBN-Nummer haben, aber im Netz kaum auffindbar sind. Die Problematik ist bekannt von Selbst-Publishing- und ähnlichen online-Verlagen, bei wissenschaftlichen Veröffentlichungen erhält sie aber noch einmal eine besondere Brisanz, da die Arbeiten auf diese Weise der Fachdiskussion weitgehend entzogen bleiben.

Das ist z.B. der Fall des oben erwähnten, 2017 an der UdeG verlegten Sammelbands (DÍAZ PÉREZ et al.) Alemán como lengua extranjera: Estudios interculturales de lengua, literatura y cultura alemanas, der Arbeiten (ehemaliger) Studenten und einiger Dozenten des 
Gruhn, D. H. - Publikationen der mexikanischen Germanistik im Wandel

an dieser Universität verankerten Masterstudiengangs enthält ${ }^{35}$. Die einzelnen Artikel sind bei einer Stichwort- oder Autorensuche in online-Suchmaschinen kaum zu finden.

\subsection{Verleger}

Wie aus Tabelle 7 bereits hervorgeht, stand hinter $46 \%$ aller Einträge des Gesamtzeitraums der Deutschlehrerverband AMPAL, der seine Publikationen selbst verlegte und herausgab. Gesponsert wurden diese in erster Linie vom Goethe-Institut in Mexiko-Stadt, der Sammelband vom DAAD. In den Jahren 2012-2014 ${ }^{36}$ konnten sich die Ausgaben 24 bis 28 der Zeitschrift infolge von Werbeeinahmen selbst finanzieren (und darüber hinaus sogar einen Gewinn für den Verband erwirtschaften). Hinter weiteren Tagungsakten (ALEG u.a.) sowie der Zeitschrift DaF-Brücke stehen ebenfalls nationale Deutschlehrerverbände bzw. deren Zusammenschlüsse, auch sie wurden/werden von Mittlerorganisationen gefördert, oftmals im Verbund.

Gemeinsam decken die vorgenannten Schriften rund 60\% aller Einträge der DaFListe ab. Zählt man noch die Titel dazu, die von der UNAM verlegt wurden (82) kommt man auf rund $75 \%$. Bezüglich der übrigen Einträge ist keine Konzentration bestimmter Verleger feststellbar.

\section{Deutsch als Publikationssprache}

Insgesamt zählten wir 138 Autoren, davon 106, also 76,8\%, mit L1 Deutsch ${ }^{37}$. Diese decken zusammen 414, d.h. 86,3\% aller Einträge der DaF-Liste ab. Vorrangige Publikationssprache im Bereich DaF ist das Deutsche, insgesamt 321, d.h. 66,9\% der Titel sind auf Deutsch verfasst, die übrigen auf Spanisch und in wenigen Ausnahmefällen in anderen Sprachen. Damit unterscheidet sich DaF wesentlich von der Germanistik i.e.S., wo die Autoren mit L1 Deutsch knapp 37\% der Einträge abdeckten und nur 13,4\% der Gesamteinträge auf Deutsch

\footnotetext{
${ }^{35}$ Mit Ausnahme von Christiane Nord, die nicht an diesem Master lehrt, sondern lediglich 2015 für einen Gastvortrag in Guadalajara eingeladen war.

${ }^{36}$ Unter dem Schatzmeister Wolfgang Kresse.

${ }^{37}$ Kriterium zur Identifizierung von L1-Sprechern war, neben persönlichem Bekanntsein der Autoren, ihr Name. Dabei ist eine Fehlerquote nicht ausgeschlossen, diese dürfte aber in jedem Fall geringfügig sein.

Pandaemonium, São Paulo, v. 24, n. 43, mai.-ago. 2021, p. 1-37
} 
Gruhn, D. H. - Publikationen der mexikanischen Germanistik im Wandel

geschrieben waren. Doch auch im DaF-Bereich haben nur 6,5\% der deutschsprachigen Titel Autoren mit L1 Spanisch.

Tabelle 9: Publikationssprache Deutsch gemäß Autoren mit L1 Deutsch oder L1 Spanisch

\begin{tabular}{|l|l|l|l|l|}
\hline \multicolumn{1}{|c|}{ Autoren } & Anzahl $^{38}$ & $\begin{array}{c}\text { Einträge } \\
\text { gesamt }\end{array}$ & $\begin{array}{c}\text { davon Einträge auf } \\
\text { Deutsch }\end{array}$ & \multicolumn{1}{|c|}{$\begin{array}{c}\text { Anteil an allen } \\
\text { deutschsprachigen Titeln } \\
(\mathrm{n}=321)\end{array}$} \\
\hline L1 Deutsch & 106 & 414 & $300(72,5 \%, \mathrm{n}=414)$ & $93,5 \%$ \\
\hline L1 Spanisch & 32 & 66 & $21(32 \%, \mathrm{n}=66)$ & $6,5 \% \%$ \\
\hline Gesamtwerte & 138 & 480 & $321(66,9 \%, \mathrm{n}=480)$ & $100 \%$ \\
\hline
\end{tabular}

Quelle: eigene Darstellung.

Die Gründe für die Dominanz des Deutschen liegen auf der Hand: Insgesamt 72,5\% der Einträge wurden in Medien veröffentlicht, in denen das Deutsche als Publikationssprache, wenn nicht verpflichtend, so zumindest erwünscht oder zugelassen ist (wie AMPAL- oder ALEG-Publikationen und sonstige Akten von Deutschlehrerkongressen, der Zeitschrift DaF-Brücke oder in Deutschland verlegten Zeitschriften oder Sammelbänden). Bezüglich der Dominanz der Autoren mit L1 Deutsch kann spekuliert werden, dass diese i.d.R. weniger Schwellenangst haben, sich mit einem Beitrag auf Deutsch zu beteiligen.

\section{Themenbereiche}

Bei der Abgrenzung der Schriften, die sich explizit mit DaF beschäftigen, von solchen der Germanistik i.e.S. treten kaum Zweifelsfälle auf, erstere nennen das Thema DaF häufig schon im Titel. Schwieriger gestaltet sich die Aufteilung innerhalb der DaF-Einträge. Es handelt sich um eine künstliche Untergliederung, denn der starke Praxisbezug des Fachbereichs bringt es mit sich, dass ein Großteil der Arbeiten einen didaktischen Zuschnitt hat. Die Themenbereiche gehen also ineinander über. Nachfolgend eine Tabelle, aus der die Untergliederung ersichtlich wird, die wir im DaF-Bereich vorgenommen haben, und die im Anschluss jeweils kommentiert wird:

\footnotetext{
${ }^{38}$ Einschließlich jeweils drei (Deutsch) und zwei (Spanisch) Personen, deren L1 weder das Deutsche noch das Spanische ist, die aber einen langjährigen Migrationshintergrund in dem entsprechenden Sprachraum aufweisen.

Pandaemonium, São Paulo, v. 24, n. 43, mai.-ago. 2021, p. 1-37
} 
Gruhn, D. H. - Publikationen der mexikanischen Germanistik im Wandel

Tabelle 10: Thematische Unterbereiche $\mathrm{DaF}$

\begin{tabular}{|l|l|l|}
\hline \multicolumn{1}{|c|}{ Unterbereich } & \multicolumn{1}{c|}{ Einträge } & \multicolumn{1}{c|}{$\mathrm{n}=480$} \\
\hline Multithematisch & 11 & $2,3 \%$ \\
\hline Lehrwerke & 28 & $5,8 \%$ \\
\hline DaF-Theorie & 34 & $7,1 \%$ \\
\hline Literaturdidaktik und Dramapädagogik & 36 & $7,5 \%$ \\
\hline Landeskunde und Interkulturalität & 83 & $17,3 \%$ \\
\hline Bericht und Ausbildung & 87 & $18,1 \%$ \\
\hline $\begin{array}{l}\text { (Angewandte) Linguistik (einschließlich Grammatik, } \\
\text { Lexik, Phonetik, Übersetzung) }\end{array}$ & 87 & $18,1 \%$ \\
\hline Didaktik/Methodik und Evaluierung & 114 & $23,8 \%$ \\
\hline Gesamt & 480 & $100 \%$ \\
\hline
\end{tabular}

Quelle: eigene Darstellung.

\subsection{Multithematische Arbeiten}

Hierunter fallen ausschließlich Herausgebertitel, insgesamt 17, von denen sechs einem spezifischen Themenbereich zugeordnet werden können. Bei den verbleibenden elf handelt es sich um die Herausgabe von acht AMPAL-Kongressakten und drei Sammelbänden. Gemeinsam ist ihnen, dass sie Texte umfassen, die mehreren DaF-Unterbereichen zugeordnet werden können.

\subsection{Lehrwerke}

Der Unterbereich „Lehrwerke“ (einschließlich Zusatzmaterialien und Arbeiten zur Lehrwerksanalyse) gehört mit 5,8\% zu den kleinen Unterbereichen. Doch darf das nicht darüber hinwegtäuschen, dass sich dahinter teils Großes verbirgt, und das nicht nur am Seitenumfang gemessen. Zeitlich setzt er im Jahr 1972 mit dem ersten mexikanischen AudioDeutschkurs Hallo Marlene. Alemán por radio para avanzados ein, ausgearbeitet von Heckel, Klier, Rall, Rall und Schur, unter der Regie von Dietrich Rall mit professionellen Sprechern im Studio von Radio UNAM aufgenommen. Marlene Rall war übrigens nicht Namensgeberin des Kurses, sondern eine Fernsehwerbung der Firma Volkswagen in Mexiko, die mit „Hallo Marlene“ begann.

Ein weiteres, noch handwerklich produziertes Mimeo von Dietrich Rall folgt 1978: Lesen will gelernt sein. Cuaderno para el alumno y Guía para el maestro; auch die 
Gruhn, D. H. - Publikationen der mexikanischen Germanistik im Wandel

Comprensión de Lectura en Alemán von Ulrike Tallowitz (1985) hat ein SchreibmaschinenLayout und enthält einige handschriftliche Korrekturen in der Druckfassung. Dieser und ein weiterer Eintrag derselben Autorin sind die einzigen (von 12), die vor dem Jahr 1997 nicht aus der Feder von Dietrich und/oder Marlene Rall als Autor(en) oder Ko-Autor(en) stammen. Danach übernimmt Siegfried Böhm die Spitzenposition mit fünf Titeln.

Inhaltlich hervorzuheben beim Themenbereich Lehrwerke ist die Häufigkeit von Arbeiten rund ums Lesen (knapp ein Viertel). Zu den jüngeren Lehrwerken zählt dabei Spaß am Lesen (2008/2013) von Siegfried Böhm (gegenwärtig in einer Neuauflage begriffen). Die Auflagenzahl liegt bei 500 Exemplaren, dieses und ähnliche Werke waren bzw. sind also vorrangig für den internen Gebrauch bestimmt, d.h. für die Lerner der Universität, die sie verlegt.

Ein größeres Verbreitungsfeld (bis hin in andere Länder) hatten die Lehrwerke Sprachbrücke I (MEBUS et al. 1987) und Sprachbrücke II (MEBUS et al. 1989) unter KoAutorschaft von Marlene Rall. Erwähnenswert ist darüber hinaus die Mitarbeit Dietrich Ralls am Mannheimer Gutachten zu ausgewählten DaF-Lehrwerken (Auswärtiges Amt der Bundesrepublik Deutschland 1977), erster Kriterienkatalog für Lehrwerksanalyse, dem später weitere folgen sollten (z.B. 1985 der Stockholmer Kriterienkatalog 1982).

\subsection{DaF-Theorie}

Darunter fallen Forschungsarbeiten und Aufsätze, die sich mit Grundfragen auseinandersetzen, die DaF tangieren. Der Übergang zur Didaktik ist teilweise fließend, Einschlusskriterium war der höhere Abstraktionsgrad. Mit 7,1\% aller DaF-Einträge handelt es sich zahlenmäßig um einen kleinen Bereich.. Die Themen sind breit gestreut, es geht vorrangig um kommunikative Kompetenz oder Kompetenzmodelle (z.B. TERBORG 2003), aber auch um das Verhältnis von Theorie und Praxis (z.B. HAUSSTEIN 2004), Qualitätssicherung (z.B. LAUERBACH 2000) oder Machtstrukturen rund um den Fremdsprachenunterricht (z.B. HASAM 1998).

Die Einträge sind relativ gleichmäßig über den Gesamtzeitraum verteilt. 58,8\% stammen von Autoren der UNAM (ohne eine Konzentration bestimmter Namen), fast ebenso

Pandaemonium, São Paulo, v. 24, n. 43, mai.-ago. 2021, p. 1-37 
Gruhn, D. H. - Publikationen der mexikanischen Germanistik im Wandel

häufig (53\%) befinden sich die Texte dieses Themenbereichs in Kongressakten. Nur 35,3\% der Titel zählen nicht mehr als sechs Seiten - weniger als der DaF-Durchschnitt (43,6\%).

\subsection{Literaturdidaktik und Dramapädagogik}

Der Unterbereich „Literaturdidaktik und Dramapädagogik“ ist mit 7,5\% der Gesamtliste ähnlich selten vertreten wie die Theorie. Beide beinhalten Unterschiedliches, trotz enger Berührungpunkte: während die Literaturdidaktik Vorschläge unterbreitet, wie mit authentischen (oder ggf. didaktisierten) i.d.R. schriftlichen literarischen Texten im DaFUnterricht gearbeitet werden kann (so z.B. HÜTTINGER 2004: „Vom Umgang mit Literatur im Fremdsprachenunterricht"), setzt die Dramapädagogik vor allem auf mündliche Improvisation zwecks Verstärkung bestimmter Kompetenzen (z.B. BoEHM 2010: „Affektiv ist effektiv. Wie dramapädagogische Lerhrformen zu interkultureller Sensibilität verhelfen sollen").

Festzuhalten sind die folgenden Eigenheiten: Nur 11 der 36 Titel wurden vor 2002 veröffentlicht, das Interesse an solchen Themen ist im neuen Jahrtausend also gestiegen. Von Autoren der UNAM stammen 63,9\% der Titel. Bei der Literaturdidaktik (23 Einträge) lässt sich bezüglich der Autoren keine namentliche Konzentration erkennen, von den 13 Texten zur Dramapädogogik stammen allerdings acht aus der Feder von Siegfried Böhm ${ }^{39}$.

Inhaltlich widmen sich die literaturdidaktischen Titel mehrheitlich allgemeinen Einführungen und Reflexionen (wie z.B. MAGER 2008: „Das Vertrautwerden mit literarischen Texten und seine Bedeutung für die Motivation der DaF-Studenten”), vereinzelt einem bestimmten Autor (Brecht, Kehlmann, Traven) oder einem Motiv (RALL 2014: „Die Farbe Blau: Literatur und Kunst im DaF-Unterricht”). Dreimal erscheinen Arbeiten zur konkreten Poesie. Auffallend ist, dass die Textsorte Comic bis auf eine Ausnahme gar nicht behandelt wird. Dabei kann diese auf Grund ihrer bildlichen Entlastung sowie umgangssprachlicher und lautmalerischer Elemente durchaus im Unterricht eingesetzt werden.

\footnotetext{
${ }^{39}$ Sein Lehrerhandbuch Aprender actuando (2013) findet sich unter den Lehrwerken.

Pandaemonium, São Paulo, v. 24, n. 43, mai.-ago. 2021, p. 1-37
} 
Gruhn, D. H. - Publikationen der mexikanischen Germanistik im Wandel

\subsection{Landeskunde und Interkulturalität}

Zwischen diesem und den vorigen Themenbereichen liegt ein quantitativer Sprung: er stellt mit 83 Einträgen, beginnend zum Jahr 1985, 17,3\% der Gesamtliste DaF, darunter ein Herausgebertitel. Lediglich 36,1\% der Titel umfassen nicht mehr als sechs Seiten, es besteht also eine Tendenz zu längeren Texten. Autoren der UNAM stellen 65\% der Titel dieses Unterbereichs und drei Autoren (Siegfried Böhm, Marlene Rall und Dietrich Rall) decken für sich allein $41 \%$ der Einträge ab. Die Beteiligung von (Orts-)Lektoren ist mit 28,9\% unterdurchschnittlich.

Weniger als als ein Drittel der Einträge (28,9\%) widmet sich der Landeskunde/ Kulturstudien im herkömmlichen Sinne. Sie thematisieren deren Bedeutung und Methoden im Fremdsprachenunterricht, beschäftigen sich mit einem bestimmten deutschsprachigen Land oder spezifischen landeskundlichen Aspekten (wie z.B. GRÄFE 2008: „Feste als Landeskundliches Thema“), häufig verbunden mit konkreten Unterrichtsvorschlägen (z.B. SADOVSKI 2010: „Kölner Karneval - ein Unterrichtsentwurf“). Das Thema kulminiert im Jahr 2017 in Form des infolge eines internationalen Kongresses an der UNAM entstandenen Sammelbandes Kulturelles Lernen im DaF/DaZ-Unterricht. Paradigmenwechsel in der Landeskunde (HAASE \& HÖLLER 2017).

Die Mehrzahl der Einträge behandelt interkulturelle Fragestellungen und trägt das Adjektiv „,interkulturell“ häufig schon im Titel (z.B. interkultureller Dialog, enfoque intercultural, insgesamt 33 mal). Zur Interkulturalität zählen wir auch Schriften zur Xenologie, Fremdwahrnehmung, Fremdheitserfahrung u. ̈̈. Der Übergang vom „Blick auf“ (traditionelle Landeskunde) und der „Interaktion zwischen" Kulturen ist dabei fließend (Beispiele: PFLEGER 2017: „Identitätskonstruktion im Fremdsprachenunterricht. Ein Pilotprojekt DaF in Mexiko“, oder WEIDEMANN 2017: „Transkulturelles Lernen mit Spielen und Lernaufgaben“), beide sind in dem eben erwähnten Sammelband enthalten.

\subsection{Bericht und Ausbildung}

Der Themenbereich „Bericht“ umfasst vorrangig Lagebeschreibungen des Deutschen als Fremdsprache in Mexiko, aber auch die Vorstellung bestimmter Institutionen, die Rolle des DAAD, binationale Wissenschaftsbeziehungen oder Arbeitstagungen im Bereich DaF. Unter 
Gruhn, D. H. - Publikationen der mexikanischen Germanistik im Wandel

„Ausbildung“ fallen Grundsatzüberlegungen zur Ausbildung von DaF-Lehrern sowie die Vorstellung spezifischer Ausbildungsprogramme oder Studiengänge.

Mit 87 Einträgen aus den Jahren zwischen 1973 und 2014 stellt dieser Bereich 18,1\% der DaF-Liste. 51,7\% der einschlägigen Titel zählen nicht mehr als sechs Seiten. 44,8\% stammen von Autoren der UNAM und knapp 90\% von Autoren mit L1 Deutsch. Es gibt 50 (Orts-)Lektoreneinträge sowie sieben Einträge von Vertretern der deutschen oder österreichischen Botschaft oder des Goethe-Instituts, zusammen stellen sie also 65,5,\% des Bereichs, woran sich das besondere Interesse der Mittlerorganisationen an solchen Fragestellungen ablesen lässt. Die sprachliche Verteilung ist hier relativ ausgewogen, 52,9\% der Titel sind auf Deutsch, 47,1\% auf Spanisch, was wiederum darauf schließen lässt, dass man auch mexikanische Vertreter von Bildungsinstitutionen und Politik erreichen wollte.

Drei Viertel der insgesamt 20 Einträge zu Fragen der Ausbildung stammen von Autoren der UNAM, eine namentliche Autorenkonzentration ist nicht zu erkennen Als Beispiel kann Bauer (2007) angeführt werden, der in seinem Artikel notwendige Komponenten einer DaF-Lehrer-Ausbildung umreißt und nachfolgend sämtliche in Mexiko existierenden oder geplanten Ausbildungsmöglichkeiten vorstellt. Bei den übrigen 67 Berichten (darunter zwei Herausgeberschriften) stellen zwei Autoren (Dietrich Rall und Martin Dettmer) für sich allein 26,9\% der Einträge. Eine Konzentration dieser Textsorte bietet der oben erwähnte AMPAL-Sammelband (AMPAL et al. 2007), in dem mehr als 15 Institutionen und Mittlerorganisationen vorgestellt werden, die in Mexiko DaF anbieten oder fördern.

\section{7 (Angewandte) Linguistik (einschließlich Grammatik, Lexik, Phonetik, Übersetzung)}

Die 87 Einträge $(18,1 \%)$ der „(Angewandten) Linguistik” umfassen die Jahre 1975 bis 2018, stammen zu 70,1\% von Autoren der UNAM und zu 75\% aus der Feder eines oder einer der oben aufgelisteten ,Vielveröffentlicher”. Veröffentlicht wurden sie überdurchschnittlich oft in Sammelbänden oder Fachzeitschriften in Deutschland (21,8\%) und unterdurchschnittlich selten in AMPAL-Medien (ebenfalls 21,8\%). Es dominieren Beiträge in Kongressakten $(40,2 \%)$ gefolgt von Zeitschriftenartikeln (28,7\%). Der Lektorenanteil liegt bei 36,8\% und somit etwas unter dem Durchschnittswert von $40 \%$.

Pandaemonium, São Paulo, v. 24, n. 43, mai.-ago. 2021, p. 1-37 
Gruhn, D. H. - Publikationen der mexikanischen Germanistik im Wandel

Inhaltlich setzen sich die Arbeiten des Bereichs u.a. mit allgemeinen Problemen der Angewandten Linguistik (24) auseinander, so zum Beispiel Bauer (2003), der die Frage aufwirft, ob Deutsch eine schwere Sprache sei.

Des Weiteren fällt darunter die Lexikologie einschließlich ihrer Didaktik, die mit 14 Titeln vor allem zur Phraseologie vertreten ist. Denn DaF-Lehrer fragen sich natürlich immer: „Wie kommen die Wörter in den Kopf?” (LAUTERBACH 2004). Weniger Aufmerksamkeit erfahren die „Übersetzung im Fremdsprachenunterricht” (6 Titel, 4 davon von Ulrike Sperr) und Phonetik (5 Titel).

Etwas ausführlicher eingegangen werden soll auf den Schwerpunkt des Unterbereichs, nämlich die Grammatik (Morphologie und Syntax), die 38 Arbeiten (43,7\%) Arbeiten stellt. Sie umfassen die Jahre 1980 bis 2017 und machen somit 7,9\% der gesamten DaF-Liste aus. Die Hälfte der Grammatik-Titel wurde vor 1994 verfasst (alle bis auf eine Ausnahme von Dietrich und/oder Marlene Rall, insgesamt liegt der Anteil beider Autoren an der Thematik bei 60,5\%). Bei den übrigen Autoren besteht keine namentliche Konzentration, auffällig ist allerdings, dass 36 der 38 Grammatik-Einträge, d.h. 95\%, von Autoren stammen, die mit der UNAM in Verbindung stehen. Grammatik-Titel wurden bevorzugt in Sammelbänden veröffentlicht (14), gefolgt von Kongressakten und Zeitschriften (jeweils 12). Auch haben wir - zwecks Wahrung der thematischen Einheit - zwei Kurzgrammatiken für Studenten in diesen Bereich einsortiert. Themen zur Grammatik wurden kaum bei AMPAL veröffentlicht (nur 4 Einträge), und ebenso selten in ALEG-Tagungsakten (5).

Die Dominanz von Marlene Rall und, in etwas geringerem Maße, Dieter Rall bei Arbeiten rund um die Grammatik rührt daher, dass sie sich intensiv mit der Dependenzgrammatik auseinandergesetzt haben. Das geschah in Zusammenarbeit mit Ulrich Engel, damaliger Direktor des Instituts für Deutsche Sprache in Mannheim. 1977 erschien in Heidelberg die Dependenz-Verb-Grammatik für Deutsch als Fremdsprache (RALL; ENGEL; RALL), 1985 neu aufgelegt, die Ausgangspunkt vieler weiterer Publikationen werden sollte (vgl. Rall \& Rall 1996: 442). Das Werk versteht sich als Pädagogische Grammatik für Lehrende und fortgeschrittene Lerner und geht (in Anlehnung an die Dependenz-Theorie von Lucien Tesnière) von einem Modell aus, in dem das Verb die anderen Wortarten regiert. Es enthält einen theoretisch-linguistischen und einen anwendungsbezogenen Teil. Auch Schüler 
Gruhn, D. H. - Publikationen der mexikanischen Germanistik im Wandel

von Marlene Rall veröffentlichten zu diesem Thema (Martha Franco; Claudia García, Rubén Garciadiego; Rocía Madrid; Laura Velasco). Nur wenige Titel der Grammatikliste sind nicht in diesem Umfeld entstanden.

\subsection{Didaktik/Methodik und Evaluierung}

„Didaktik/Methodik und Evaluierung“ stellen mit 23,8\% aller Titel das größte Kontingent der DaF-Liste und verteilen sich über fast den gesamten Erhebungszeitraum (1972 bis 2017). Allerdings mit erheblichen zeitlichen Verschiebungen: 30,7\% der Titel des Themenbereichs erschienen vor dem Jahr 2000 (1,3 /Jahr), 45,6\% allein in dem Jahrzehnt zwischen 2000 und 2009 (5,2 /Jahr) und 25,4\% ab 2010 (3,2/Jahr). Was die Institutionszugehörigkeit betrifft, so stammen 43,9\% aller Einträge des Bereichs von Autoren der UNAM, diese sind somit ausnahmsweise unterrepräsentiert (Schnitt 57,5\%), was bedeutet, dass die Thematik verstärkt auch Autoren anderer Institutionen zum Schreiben motiviert. Nächststarke Gruppen sind Autoren der UdeG mit 15,8\%, der UNACH in Chiapas, 7\%, und des Goethe-Instituts, 6,1\%; darüber hinaus sind keine Häufungen zu beobachten. Auch namentlich konzentrieren sich einzelne Autoren hier nicht so stark wie bei anderen Themenbereichen: Spitzenreiterin ist Katharina Herzig, UdeG, mit 7,9\% der Einträge, gefolgt von Martin Dettmer, UNACH, 6,1\%, Siegfried Böhm, UNAM (FES-Acatlán), 5,3\%, und Marlene Rall, UNAM, 4,4\%.

Was die Publikationsmedien betrifft, so liegt der Themenbereich sowohl bei den Kongressakten $(55,3 \%)$ als auch bei den Zeitschriftenartikeln $(41,2 \%)$ deutlich über den Durchschnittswerten von jeweils 42,9\% und 32,7\%, bei den Beiträgen in Sammelbänden mit 3,5\% jedoch wesentlich darunter (Durchschnitt sind 19,4\%). Das wiederum legt den Schluss nahe, dass zu diesem Themenkomplex vorrangig bei AMPAL publiziert wurde. Eine Überprüfung bestätigt das: 40,4\% der Titel erschienen in AMPAL-Kongressakten, 24,6\% bei info-ampal. Zählt man noch die neun Beiträge in ALEG-Kongressakten und die vier in der Zeitschrift DaF-Brücke dazu, so umfassen diese vier Medien insgesamt 76,3\% aller Einträge unter „Didaktik/Methodik und Evaluierung“.

Bezüglich der Seitenzahl liegt der Themenbereich im Durchschnitt: 40,4\% der Einträge haben bis zu sechs Seiten (Schnitt der Gesamtliste DaF 43,1\%).

Pandaemonium, São Paulo, v. 24, n. 43, mai.-ago. 2021, p. 1-37 
Gruhn, D. H. - Publikationen der mexikanischen Germanistik im Wandel

Die Inhalte von „Didaktik und Methodik“ sind relativ breit gefächert. Die meisten Einträge (19,3\%) stellen Arbeiten zur Leistungsmessung und -beurteilung, einschließlich Fehleranalyse und -bewertung, Evaluierungsformen, Prüfungsbeschreibungen u.a. (z.B. HiRSCHFELD 2003). Bis auf drei Ausnahmen stammen alle aus diesem Jahrtausend.

Im 21. Jahrundert verortet (nur drei Ausnahmen) sind auch die meisten Arbeiten rund um elektronische Unterrichtsmedien (insgesamt 16,7\% des Themenbereichs), darunter fallen z.B.: E-Learning, Blended-Learning, Nutzung des Internet, elektronische Selbstlernmaterialien, aber auch der Einsatz von Filmen im Unterricht (z.B. LISZT 2014). Bei einigen Texten handelt es sich um allgemeine Einführungen, andere unterbreiten Unterrichtsvorschläge anhand eines bestimmten Materials.

Ein weiterer gewichtiger Themenkomplex sind die Lehr- und Lernformen (15,8\%), das umfasst nach Rösler (2012: 95-125) u.a. die Lernerautonomie - ein Thema das zwischen 1998 und 2008 Hochkonjunktur hatte, auch im Zusammenhang mit Lernstrategien. Ebenfalls unter diese Rubrik fallen Texte zu Sozialformen des Unterrichts, wie z.B. kooperativer Unterricht, aber auch zu bestimmten Arbeits- oder Übungsformen wie etwa Portfolio-Arbeit oder der Einsatz von Spielen im Unterricht (z.B. Au 2004).

An vierter Stelle stehen die rezeptiven und produktiven Fertigkeiten (14,9\%), die einschlägigen Arbeiten verteilen sich über den gesamten Erhebungszeitraum. Dem „Lesen“ fällt dabei die größte Aufmerksamkeit zu (z.B. HERZIG 2012a), gefolgt vom „Schreiben“. Die Fertigkeit „Hören“ kommt auf zwei Einträge und nur ein Eintrag widmet sich explizit der Entwicklung der „Sprechfertigkeit“ (wobei diese sicher von anderen Bereichen, wie z.B. der Dramapädagogik, mit tangiert wird).

Im Rahmen des Sachgebiets „Lernende und Lehrende“ (vgl. RÖSLER 2012: 5-16), 13,2\%, wurde zu den affektiven Faktoren auf Seiten der Lerner veröffentlicht, am häufigsten (9 mal) zur „Motivation“ (z.B. NEUDECKER; EGLI 2004), aber auch zur Emotionalität. Ebenso fand die Kreativität der Lehrer Beachtung, deren Aufgabe darüber hinaus die Unterrichtsplanung ist, der sich drei Titel widmen.

Sieben Titel $(6,1 \%)$ umreißen die Fremdsprachendidaktik allgemein (z.B. BAUER 2008) oder umfassen mehrere Themenfelder (z.B. die Kongressakten des ALEG XIV, HERZIG et al. 2014).

Pandaemonium, São Paulo, v. 24, n. 43, mai.-ago. 2021, p. 1-37 
Gruhn, D. H. - Publikationen der mexikanischen Germanistik im Wandel

Bei ebenfalls sieben Einträgen (6,1\%) handelt es sich um Forschungsbeschreibungen. Wir haben sie auf Grund ihrer engen Anbindung an die Praxis mit zur Didaktik gestellt (statt zur Theorie). Es geht vorrangig um Unterrichtsbeobachtung sowie um regional- und zielgruppenspezifischen DaF-Unterricht (z.B. HERZIG 2014 und 2012b).

Einige wenige Titel widmen sich zwischen 1972 und 1997 der Fachsprachenvermittlung ${ }^{40}$, ein Thema, das danach nie wieder aufgenommen wurde. Dabei gäbe es gerade hier einen großen Forschungs- und methodischen Enwicklungsbedarf, nicht zuletzt, weil es die deutschsprachigen Länder selbst sind, die ausländische Fachkräfte in bestimmten Arbeitsfeldern intensiv nachfragen. Hier ist vor allem an IT-Spezialisten und den medizinischen Bereich zu denken. Spezielle Unternehmen heuern gezielt mexikanische Krankenschwestern und -pfleger für Deutschland an, ein Beispiel im Bereich der Altenpflege ist die Firma Concura (https://concura.mx/). Einige deutsche Verlage haben fachspezifische Lehrbücher ab dem Niveau B1 auf den Markt gebracht, so z.B. der Hueber Verlag: Menschen im Beruf-Pflege B1 (Hagner 2016). Mit Ausnahme eines Angebots des Goethe-Instituts in Mexiko Stadt ab der Stufe B2 (,Deutsch für medizinisches Personal“) gibt es in Mexiko bislang keine auf diese Zielgruppe zugeschnittenen Sprachkurse, geschweige denn einschlägige Forschungsarbeiten.

\section{Ausblick}

Die Datenauswertung hat gezeigt, dass die DaF-Publikationen der mexikanischen Germanistik in ihren Grundlagen von wenigen Autoren eingeleitet und getragen wurden, wobei die UNAM sowie DAAD-Lektoren eine herausragende Stellung einnahmen. Mit dem weitgehenden Abgang der frühen Autoren, dem Wegfall der AMPAL-Publikationsmedien (fast die Hälfte aller Einträge der Liste) und die vor wenigen Jahren erfolgte Einstellung der Fördermittel für Publikationen seitens des DAAD ist das ehemals rege

\footnotetext{
${ }^{40}$ In den 70er und 80er Jahren beschäftigte sich eine fach- und institutionenübergreifende Forschergruppe in Mexiko-Stadt intensiv mit dem Thema Leseverstehen von Fachtexten. Hervorgegangen sind daraus die Titel Meyer-Minnemann; Rall (1972), die vom DAAD verlegte und von Dietrich Rall und Heinz Schepping herausgegebenen Akten einer Arbeitstagung in Aachen (1976), in der Dietrich Rall auch einen Artikel einfügt (beide unter "Berichten" in dieser Arbeit) sowie ein Titel von Ulrike Tallowitz (1989), den sie 1997 zusammen mit Christian Fandrych noch einmal aufgegriffen und erweitert hat. Weitere in diesem Rahmen entstandene Titel wurden dem Bereich "Lesen" zugeordnet.

Pandaemonium, São Paulo, v. 24, n. 43, mai.-ago. 2021, p. 1-37
} 


\section{Gruhn, D. H. - Publikationen der mexikanischen Germanistik im Wandel}

Publikationsaufkommen seit 2010, und besonders seit 2015, auf einen Bruchteil früherer Werte zusammengeschrumpft. Dass diese Tendenz unter den gegenwärtigen Bedingungen rückgängig gemacht werden kann, muss bezweifelt werden.

$\mathrm{Zu}$ bedauern ist das allemal. Führt man sich vor Augen, dass insbesondere die Veröffentlichungen des Deutschlehrerverbands AMPAL nicht in erster Linie der wissenschaftlichen Erkenntniserweiterung dienten, sondern einen engen Praxisbezug hatten, so wird deutlich, dass diesen, neben ihrem informativen Wert, eine wichtige integrierende Funktion zukam. Sie dienten als Einstiegs- und Ausdrucksmedium für Deutschlehrer in Mexiko, vielen boten sie die Möglichkeit, überhaupt zu publizieren, Teil der mexikanischen DaF-Öffentlichkeit zu sein und sich dort zu orientieren. Einschlägige Online-Foren könnten diese Funktionen teilweise übernehmen, aber sicher nicht vollständig ersetzen. Im Moment zeichnet sich allerdings kein solches Forum für regionale Belange ab.

Darüber hinaus schwächt sich mit dem Rückgang von Publikationen wissenschaftlich orientierter Autoren nicht nur die Rolle solcher Experten als potenzielle Vorbilder und Zugpferde für die DaF-Forschung und -Lehre in Mexiko, sondern auch die Strahlkraft der mexikanischen Germanistik und DaFschaft schlechthin. Die Gründe, warum diese einst vorhandenen Kapazitäten nicht in gleichbleibender Intensität erhalten werden konnten, sind sicher vielfältig und komplex, im Rahmen der hier vorliegenden Bestandsaufnahme können sie nicht durchleuchtet werden. Anzumerken sei jedoch, dass der Sprachlehrforschung heute allgemein wenig Aufmerksamkeit gewidmet wird. Im bildungspolitischen Interesse stehen kostengünstige Lehrangebote mit schwachem akademischen Unterbau sowie der massive Personenaustausch zwischen Universitäten verschiedener Länder, dessen Hauptzweck mitunter in der Erfüllung oder Überbietung von Soll-Quoten zu liegen scheint, zumindest bis zur Corona-Pandemie.

Eine Lehre lässt sich aus der Gesamtstudie auch insofern ziehen, als sie deutlich macht, wie sehr erfolgreiche Entwicklungen im akademischen Bereich von nur ganz wenigen engagierten Persönlichkeiten abhängen können. Insofern meinen wir, dass sich die Universitätspolitik in Mexiko gegenwärtig auf dem falschen Weg befindet: Statt von den Akademikern zu fordern, sich als Allrounder zu verausgaben und ihre Kraft und Zeit auf die 
Gruhn, D. H. - Publikationen der mexikanischen Germanistik im Wandel

unterschiedlichsten Bereiche zu verteilen (Lehre, Verwaltung, Mentoring ${ }^{41}$ u.a.), wobei der administrative Aufwand ständig höher geschraubt wird, wäre es sinnvoller, einzelnen dort den Rücken zu stärken, wo sie am leistungsfähigsten sind. Das trüge nicht nur dazu bei, die schöpferischen Potenziale der Autoren zu stärken, sondern auch die der Institutionen als solcher. Und vielleicht auch dazu, den mexikanischen DaF-Publikationen wieder zu mehr Aufwind zu verhelfen.

\section{Literaturverzeichnis}

AMPAL; DAAD; UnIVERSIDAD AUTÓNOMA DE BAJA CALIFORNIA (Hg.). El idioma alemán en México. Medio de enlace, oportunidades y prestigio. Hacia un futuro trilingüe. Mexiko, 2007.

AU, Alexander. Autonomes Lernen und traditionelle Lerngesellschaften. In: AMPAL (Hg.). VI Encuenro AMPAL 2003, 2004, 95-108.

AUSWÄRTIGES AMT DER BUNDESREPUBLIK DEUTSCHLAND. Mannheimer Gutachten zu ausgewählten DaF-Lehrwerken. Julius Groos Verlag Heidelberg, 1977.

BAUER, Ulrich. Was ist eigentlich Didaktik? In: AMPAL (Hg.). VII und VIII Memorias AMPAL 2005/2007, 2008, 78-85.

BAUER, Ulrich. Profesionalismo y éxito en la enseñanza del alemán en México. In: AMPAL; DAAD; Universidad AutónOMA De BAJA CALIFORNia (Hg.). El idioma alemán en México. Medio de enlace, oportunidades y prestigio. Hacia un futuro trilingüe. Mexiko, 2007, 29-40.

BAUER, Ulrich. Ist Deutsch eine schwere Sprache? Linguistische Bemerkungen zur typologischen Distanz Deutsch-Spanisch und der vorwissenschaftlichen Beurteilung des Deutschen. In: DaF-Brücke, n. 5, 2003, 4-7.

BERNSTEIN, Nils; LERCHNER, Charlotte. Ästhetisches Lernen im DaF-/DaZ-Unterricht. LiteraturTheater-Bildende Kunst-Musik-Film. Materialien Deutsch als Fremdsprache Band 93. Universitätsverlag Göttingen, 2014.

BoEHM, Siegfried. Aprender actuando. UNAM, FES Acatlán, 2013.

BOEHM, Siegfried. Affektiv ist effektiv. Wie dramapädagogische Lehrformen zu interkultureller Sensibilität verhelfen sollen. In: AMPAL (Hg.). IX Encuentro AMPAL 2009, DACH-Tage 2008, 2010, 70-76.

BoEHM, Siegfried. Spaß am Lesen. UNAM, FES Acatlán, 12008/22013.

DÍAZ PÉREZ, Olivia C.; FANDRYCH, Christian; RAMÍREZ BARBA, Norberto; TsCHIRNER, Erwin (Hg.). Alemán como Lengua Extranjera: Estudios interculturales de lengua, literatura y cultura alemanas. Guadalajara: Centro Universitario de Ciencias Sociales y Humanidades, Universidad de Guadalajara, 2017a.

41 Darunter ist die Betreuung einer Studentengeneration in verwaltungstechnischen und anderen Angelegenheiten zu verstehen.

Pandaemonium, São Paulo, v. 24, n. 43, mai.-ago. 2021, p. 1-37 
Gruhn, D. H. - Publikationen der mexikanischen Germanistik im Wandel

DíAZ PÉREZ, Olivia C.; GuTJAHR, Ortrud; SiguAN, Marisa (Hg.). Deutsche Gegenwarten in Literatur und Film. Tendenzen nach 1989 in exemplarischen Analysen. Tübingen: Stauffenburg Colloquium 84, 2017b.

GARCIADIEGO, Rubén. La enseñanza del alemán en el CELE de la UNAM. In: AMPAL; DAAD; UniversidAd AutónOMA de BAJA CALIFORNIA. El idioma alemán en México. Medio de enlace, oportunidades y prestigio, 2007, 45-49.

GRÄFE, Florian. Germanistik und Deutsch als Fremdsprache in Mexiko. In: MIDDEKE, Annegret (Hg.). Entwicklungstendenzen germanistischer Studiengänge im Ausland. Sprache Philologie - Berufsbezug. Göttingen: Universitätsverlag Göttingen, 2010, 77-82.

GRÄFE, Florian. Feste als Landeskundliches Thema. In: info-ampal, n. 18, 2008, 11-15.

GRUHN, Dorit Heike; HAHN, Josefin. Publikationen der mexikanischen Germanistik im Wandel: Literatur, Linguistik, Übersetzung, Landesstudien und Alterität. In: Germanistik in Lateinamerika: Entwicklungen und Tendenzen. Göttingen: Universitätsverlag Göttingen. Sammelband in Vorbereitung.

HAASE, Peter; HÖLlER, Michaela (Hg.). Kulturelles Lernen im DaF/DaZ-Unterricht. Paradigmenwechsel in der Landeskunde. Materialien Deutsch als Fremdsprache, Bd. 96. Göttingen: Universitätsverlag Göttingen, 2017.

HAGNER, Valesca. Menschen im Beruf-Pflege B1. München: Hueber Verlag, 2016.

HASAM, Stephen. El poder, la dominación y la enseñanza de lenguas extranjeras hegemónicas en regiones subordinadas. In: PROVENZAL, Alma Ortìz (Hg). Antologìa $9^{\circ}$ Encuentro Nacional de Profesores de Lenguas, CELE, UNAM, 1998, 341-350.

HAusstein, Alexandra. Die gewünschte, unmögliche Einheit von Theorie und Praxis in der Sprachvermittlumg. In: info-ampal, n. 10, 2004, 17-21.

HECKEL, Ilse. Articulación y sonido de la lengua alemana. Mexiko: UNAM, 1992.

HECKEL, Ilse. Juego escénico, bases para una nueva didáctica de lenguas extranjeras. Mexiko: UNAM, 1984.

HeCKel, Ilse; KlIER, Linde; RALl, Dietrich; RALl, Marlene; SCHUR, H.P. Hallo Marlene. Alemán por radio para avanzados. Radiokurs. UNAM, 1972.

HEMING, Ursula. El alemán en el Instituto Politécnico Nacional -retrospectiva, situación actual y perspectivas-. In: AMPAL; DAAD; UNIVERSIDAD AUTÓNOMA DE BAJA CALIFORNIA. $E l$ idioma alemán en México. Medio de enlace, oportunidades y prestigio, 2007, 51-55.

Herzig, Katharina; Pfleger, Sabine; PuPP SPINASSÉ, Karen; SADOwSKI, Sabrina (Hg.). Transformationen: DaF-Didaktik in Lateinamerika. Impulse aus Forschung und Unterrichtspraxis. Bd. 4. ALEG XIV, Guadalajara: Stauffenburg Verlag, 2014.

HERZIG, Katharina. Zielgruppen- und standortspezifischer DaF-Unterricht mit mexikanischen Studierenden: Handlungszielorientierung. In: HeRzIG, Katharina; PFLEgER, Sabine; PuPP SPINASSÉ, Karen; SADOWSKI, Sabrina (Hg.). Transformationen: DaF-Didaktik in Lateinamerika. Impulse aus Forschung und Unterrichtspraxis. ALEG XIV, Guadal./Bd. 4. Stauffenburg Verlag, 2014, 43-60.

HERZIG, Katharina. Analysekriterien für Aufgabenformate zum Leseverstehen im DaF-Unterricht. In: info-ampal, n. 24, 2012a, 13-17.

HERZIG, Katharina. Unterrichtsbeobachtung im DaF-Unterricht. In: DaF-Brücke, n. 12, 2012b, 1723.

HIRSCHFELD, Diana. Lehren und Prüfen - das ewige Problem. In: info-ampal, n. 9, 2003, 10-11.

HÜTTINGER, Christine. Vom Umgang mit Literatur im Fremdsprachenunterricht. In: info-ampal, n. $10,2004,15-17$.

Pandaemonium, São Paulo, v. 24, n. 43, mai.-ago. 2021, p. 1-37 
Gruhn, D. H. - Publikationen der mexikanischen Germanistik im Wandel

KRUMM, Hans-Jürgen et al. (Hg.). Deutsch als Fremd- und Zweitsprache: ein internationales Handbuch. 2. Halbband. Berlin/New York: De Gruyter, 2010.

LAUTERBACH, Stefan. Wie kommen die Wörter in den Kopf? In: AMPAL (Hg.). VI Encuentro AMPAL, 2004, 63-94.

LAUTERBACH, Stefan. Qualitatssicherung im Fremdsprachenunterricht. In: AMPAL (Hg.). IV Encuentro ampal, México, D.F., 2000, 8-22.

LISZT, Malgorzata. Einsatz der Web 2.0-Anwendungen im DaF-Unterricht. In: info-ampal, n. 28, 2014, 10-14.

MAdrid Hevia Del Puerto, Rocío; ZiRPIns, Roland. Die Lautlehre des Deutschen. Mexiko: CENLEX, Instituto Politécnico Nacional, 1988.

MAGER, Elisabeth. Das Vertrautwerden mit literarischen Texten und seine Bedeutung für die Motivation der DaF-Studenten. In: AMPAL (Hg.). Memorias VII Encuentro AMPAL, VIII Encuentro AMPAL, 2008, 31-38.

Mebus, Gudula; PAUldRACH, Andreas; RALl, Marlene; RöSLER, Dietmar. Sprachbrücke II. München: Klett-Verlag, 1989.

Mebus, Gudula; PAuldrach, Andreas; RAll, Marlene; RöSler, Dietmar. Sprachbrücke I. München: Klett Verlag, 1987.

MEYER-MinNEMANN, Klaus; RALL, Dietrich. Der Erwerb von Lesekenntnissen in Wissenschaftsdeutsch. Versuch mit einer Untergruppe des fachbezogenen Fremdsprachenunterrichts. In: Zielsprache Deutsch, n. 4, 1972, 168-183.

NEUDECKER, Karin; EGLI, Hans. Analysekriterien für Aufgabenformate zum Leseverstehen im DaFUnterricht. In: AMPAL (Hg.): VI Encuentro AMPAL 2003, 2004, 141-148.

Oeste DE BopP, Marianne. Die Deutschen in Mexiko. In: FröSCHLE, Hartmut (Hg.). Die Deutschen in Lateinamerika. Schicksal und Leistung. Tübingen und Basel: Horst Edelman Verlag, 1979, 475-564.

OESTE DE BOPP, Marianne. El idioma alemán en México. In: Filosofía y Letras, v. 55-56, 1954, 161177.

Pabisch, Peter; Bopp, Peter. Marianne Oeste de Bopp. In: SPAleK, John M.; FeIlchenfeldT, Konrad; HAWRYlChaK, Sandra H. (Hg.). Deutschsprachige Exilliteratur seit 1933. Berlin/New York: De Gruyter, 2010, 208-231.

PFLEGER, Sabine. Identitätskonstruktion im Fremdsprachenunterricht. In: HAASE \& HÖLLER (Hg.). Kulturelles Lernen im DaF/DaZ-Unterricht. Paradigmenwechsel in der Landeskunde. Universitätsverlag Göttingen, 2017, 25-39.

RALL, Dietrich. Die Farbe Blau: Literatur und Kunst im DaF-Unterricht. In: BERNSTEIN \& LERCHNER (Hg.). Ästhetisches Lernen im DaF-/DaZ-Unterricht. Literatur-Theater-Bildende KunstMusik-Film. Universitätsverlag Göttingen, 2014, 91-105.

RALl, Dietrich; RALL, Marlene (Hg.). Actas del VIII Congreso de Estudios Germanísticos. Mexiko: UNAM, 1996.

RALL, Dietrich; RALL, Marlene. Lesen will gelernt....sein. Cuaderno para el alumno y Guía para el maestro. Mimeo. UNAM, 1978.

RALL, Dietrich; SCHEPPING, Heinz (Hg.). Didaktik der Fachsprache. Beiträge zu einer Arbeitstagung an der RWTH Aachen vom 30.9.-4.10.1974. DAAD, 1976.

RALl, Marlene; RaLl, Dietrich (Hg.). Bibliografía de Estudios Germanísticos en México. 19491994. Mexiko Stadt: UNAM, 1994.

RAll, Marlene; ENGEL, Ulrich; RALl, Dietrich. Dependenz-Verb-Grammatik für Deutsch als Fremdsprache. Heidelberg: Groos, 1977.

Pandaemonium, São Paulo, v. 24, n. 43, mai.-ago. 2021, p. 1-37 
Gruhn, D. H. - Publikationen der mexikanischen Germanistik im Wandel

RAUCHBAUER, Martin. La política austriaca de las lenguas en México. In: AMPAL; DAAD; UnIVERSIDAD AUTÓNOMA DE BAJA CALIFORNIA. El idioma alemán en México. Medio de enlace, oportunidades y prestigio, 2007, 5-10.

REYEs OROZCO, Carlos. 20 Kleine Szenen für das Klassenzimmer. Übers. Ilse Heckel, 1971.

RICK, Markus. El Goethe-Institut Mexiko: 40 años laborando a favor del idioma alemán en México. In: AMPAL; DAAD; UNIVERSIDAD AUTÓNOMA DE BAJA CALIFORNIA (Hg.). El idioma alemán en México. Medio de enlace, oportunidades y prestigio, 2007, 21-23.

RÖSLER, Dietmar. Deutsch als Fremdsprache. Eine Einführung. Stuttgart-Weimar: J.B. Metzler, 2012.

SADOVSKI, Sabrina. Kölner Karneval - ein Unterrichtsentwurf. In: info-ampal, n. 23, 2010, 5-7.

SPITTA, Arnold. El fomento de la lengua alemana por el DAAD en México. In: AMPAL; DAAD; UNIVERSIDAD AUTÓNOMA DE BAJA CALIFORNIA (Hg.). El idioma alemán en México. Medio de enlace, oportunidades y prestigio, 2007, 17-20.

TALLOWITZ, Ulrike. Curso de comprensión de lectura 1. UNAM, 1985.

TALlOWITZ, Ulrike. Bausteine Fachdeutsch für Wissenschaftler: Linguistik. In: FuHR, Gerhard (Hg.): Bausteine Fachdeutsch für Wissenschaftler 1989, 447-451,

TALlOWITZ, Ulrike; FANDRYCH, Christian. Bausteine Fachdeutsch für Wissenschaftler: Linguistik. In: InfoDaf, n. 3, 1997, 480-492.

TERBORG, Roland. Kompetenz als Maßstab: Die negativen Auswirkungen. In: info-ampal, n. 8, 2003, 4-7.

VASEN, Federico; LUJANO VILCHIS, Ivonne. Sistemas nacionales de clasificación de revistas científicas en América Latina: tendencias recientes e implicaciones para la evaluación académica en ciencias sociales. In: Revista Mexicana de Ciencias Políticas y Sociales, v. 62, 231, 2017. Online: Www.scielo.org.mx/scielo.php?pid=S018519182017000300199\&script=sci_arttext (Stand: 15.07.2020).

WeIdemann, Christina. Transkulturelles Lernen mit Spielen und Lernaufgaben. In: HAASE \& HÖLlER (Hg.). Kulturelles Lernen im DaF/DaZ-Unterricht. Paradigmenwechsel in der Landeskunde. Universitätsverlag Göttingen, 2017, 289-306.

Recebido em 8 de agosto de 2020

Aceito em 25 de setembro de 2020

Pandaemonium, São Paulo, v. 24, n. 43, mai.-ago. 2021, p. 1-37 